\title{
Adaptive Interference Management of OFDMA Femtocells for Co-Channel Deployment
}

\author{
Ji-Hoon Yun, Member, IEEE, and Kang G. Shin, Fellow, IEEE
}

\begin{abstract}
Femtocell technology has been drawing considerable attention as a cost-effective means of extending cellular coverage and enhancing capacity as well as realizing its potential, when combined with orthogonal frequency-division multiple access (OFDMA), for improved indoor broadband wireless services. However, under the expected co-channel deployment of femtoand macro-cells, femtocells may incur high uplink interference to macrocells, and vice versa. To mitigate this interference, we propose a distributed and self-organizing femtocell management architecture, called the Complementary TRi-control Loops (CTRL), that consists of three control loops to determine (1) maximum transmit power of femtocell users based on the fedback macrocell load margin for protection of the macrocell uplink communications; (2) target signal to interference plus noise ratios (SINRs) of femtocell users to reach a Nash equilibrium; and (3) instantaneous transmit power of femtocell users to achieve the target SINRs against bursty interference from other nearby users. CTRL requires neither special hardware nor change to the radio resource management (RRM) of existing macrocells, thus facilitating non-disruptive (hence seamless) penetration of femtocells. Also, CTRL guarantees convergence in the presence of environmental changes and delayed feedback. Our evaluation has shown CTRL to successfully preserve the macrocell users' service quality under highly dynamic user transmission conditions and be able to make a tradeoff between macrocell and femtocell capacities.
\end{abstract}

Index Terms-Macro- and femto-cells, co-channel deployment, interference mitigation, feedback control

\section{INTRODUCTION}

$\mathbf{G}$ ROWING demands for bandwidth-hungry data services in wireless cellular networks have been a great impetus to the development of new ways of improving system capacity. Femtocell technology has emerged as a cost-effective means to improve such broadband wireless services within a home or an enterprise environment [1]. A significant capacity improvement can be achieved by the femtocell's enhanced spatial reuse of spectrum resources. A further improvement can be made by combining femtocell technology with the orthogonal frequency-division multiple access (OFDMA) technology [2] [3].

A femtocell is a small indoor coverage area under a lowpower base station (BS), referred to as a femto $B S$ in this paper. Unlike macro BSs, femto BSs are installed on the subscriber's

Manuscript received 1 June 2010; revised 1 December 2010.

Ji-Hoon Yun is with the Department of Computer Software Engineering at The Kumoh National Institute of Technology, Gumi, Gyeongbuk 730-701, Korea (e-mail: jhyun@kumoh.ac.kr).

Kang G. Shin is with the Real-Time Computing Laboratory in the Department of Electrical Engineering and Computer Science at The University of Michigan, Ann Arbor, MI 48109-2122, U.S.A. (e-mail: kgshin@eecs.umich.edu).

Digital Object Identifier 10.1109/JSAC.2011.110610.

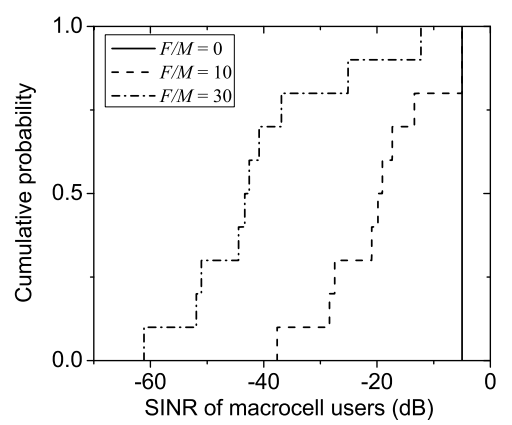

(a) Macrocell users' SINR under a varying number of femtocells

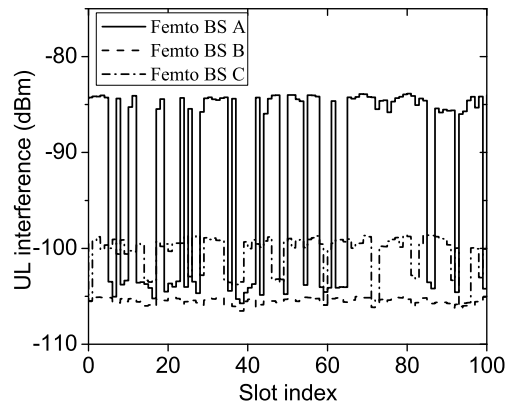

(b) UL interference evolution samples at three different femto BSs

Fig. 1. Illustration of the femtocell uplink (UL) interference problem

premise and typically connected to an operator's core network via public Internet connections (e.g., digital subscriber line (DSL) or cable modem). Femtocells benefit both subscribers and operators: better service coverage and higher indoor data throughput for subscribers, macrocell offloading and indoor coverage improvement at low capital and operational costs for operators.

\section{A. Need for Femtocell Management for Uplink Communica- tions}

A main challenge associated with the femtocell technology is how to protect-when macro- and femto-cells use the same frequency band-macrocell user services against interference from femtocell users (users being served by femtocells) while exploiting as high spatial reuse of spectrum resources as possible within each femtocell. Due to the high cost of licensed spectrum, operators may allocate femtocells the same frequency band as macrocells, called co-channel deployment. Under the expected co-channel femtocell deployment, however, transmissions of femtocell users may cause 
interference to the ongoing transmissions of users being served by macrocells, and vice versa. Several researchers [4]-[7] reported such a phenomenon and the resultant performance degradation of both macrocell and femtocell users in uplink (UL) communications as a serious problem. The femtocell UL interference was also observed in our two-tier multi-cell simulations under a realistic environmental configuration (as detailed in Section V.B). Fig. 1 shows the macrocell users' UL performance degradation due to randomly deployed femtocells. In the absence of femtocell users, all macrocell users meet the target signal to interference plus noise ratio (SINR) of $-5 \mathrm{~dB}$. As the number of femtocells within a macrocell (denoted by $F / M$ in the figure) increases, the macrocell users' achieved SINRs get deteriorated significantly. The increasing importance of UL communications for emerging interactive and content uploading services calls for a resolution of the femtocell interference problem in UL communications.

Possible UL interference scenarios between macro- and femto-cells can be illustrated as follows. Due to unplanned deployment, some femtocells could reside close to a macro BS and their users will incur high UL interference to the macro BS. The opposite also happens when a macrocell user(s) resides in the vicinity of a femto BS. To make matters worse, the users of the femto BS in such a situation get to use stronger transmit power to maintain the receive SINR and thus incur higher UL interference to the macrocells around them. A macrocell user induces higher interference to nearby femto BSs as it is farther away from its serving macrocell. Another possible interference source to femtocell's UL is neighboring femtocells. Unplanned deployment could cause multiple femtocells to be placed in proximity, possibly resulting in severe performance degradation without their proper coordination. On the other hand, unlike the UL interference to a macro BS, that to a femto BS may result from a small (if any) number of dominant wireless sources, and thus, the interference pattern could be bursty. The samples of UL interference evolution at femto BSs obtained from our simulation show such a phenomenon as illustrated in Fig. 1(b). The degree of burstiness differs for different femto BSs, depending on the presence of nearby macrocell or femtocell users. The usual power control schemes are not designed to cope with this bursty interference situation [4].

Therefore, without well-designed femtocell management, both macro- and femto-cell users will experience performance deterioration. Based on these observations, the objectives of femtocell management can be summarized as follows:

- Protection of macrocell's UL against femtocell interference;

- Efficient resource-usage coordination among femtocells; and

- Protection of femtocell's UL against bursty interference.

In general, femtocells play a supplementary role in cellular networks. Hence, we assume that the first objective is given the highest priority.

\section{B. Requirements of Femtocell Management}

Due to overlaid deployment on existing cellular networks and other salient features of femtocells, one needs to consider the following requirements when designing a femtocell management architecture.

- No change to macrocell radio resource management $(\mathrm{RRM}):{ }^{1}$ considering the fact that femtocells are generally deployed on an already-existing and working cellular infrastructure, ${ }^{2}$ a femtocell management architecture must be implemented without disrupting macrocell networks and services. This can be achieved by keeping intact performance-critical parts of macro BSs, especially RRM. The RRM within existing macrocells has been optimized and verified in the field for providing various types of user service under a wide range of cell conditions, such as traffic patterns, user population, quality-ofservice (QoS), user mobility, etc. Therefore, changing the macrocell RRM may affect already-stabilized macrocell user services and trigger tedious, costly optimization processes, and moreover, service disruptions.

- Distributed and self-organizing operation: due to user installation, unplanned deployment and possibly a large number of femtocells, femtocell management should be distributed and self-organizing. Thus, convergence using the least amount of global information in the presence of time-varying and unpredictable environmental changes becomes an important design requirement. These features along with restricted access make the femtocell problem very different from the classical hierarchical cell coordination problem [8].

- Support of legacy user devices: is important for market penetration. To achieve this, a management architecture should not define any new operation for user devices.

- No special hardware: for cost-efficiency, a femto BS should not require any special hardware.

\section{Limitations of Existing Solutions}

There have been several proposals for resolving the femtocell interference problem in downlink (DL), mainly focusing on how to avoid excessive DL interference to macrocell users in the vicinity of a femto BS. Power adaptation is to let a femto BS use as small DL transmit power as possible while serving its users. It is shown in [9] that such a scheme mitigates femtocell interference to macrocell users in DL. Femtocell sectorization radiates radio frequency (RF) energy only within sectors with users and thus reduces the possibility that nearby macrocell users are interfered with. It requires a sectorized antenna and multiple radio paths, one for each sector. Similarly, beamforming is also effective, but requires additional hardware. The above schemes operate based on the femto BS's local information only and regardless of the macrocell UL status. Therefore, they are ineffective against the UL interference problem. Although femtocell sectorization may reduce the required UL transmit power of femtocell users thanks to directional interference reception at the serving femto BS, UL interference from femtocells to macrocells is

\footnotetext{
${ }^{1}$ The priority of this requirement may, in practice, depend on the operator's policy.

${ }^{2}$ In general, macrocells are deployed first since nationwide connectivity is crucial for cellular services. For example, many operators in various countries plan to start commercial LTE macrocell services within the next few years, but mostly without any firm schedule for femtocell services.
} 
still uncontrollable and protection of macrocell's UL is not assured.

There have been a few proposals to tackle the UL problem, but they do not meet all of the requirements or objectives discussed earlier. Chandrasekhar et al. [6] proposed a noncooperative game-theoretic UL power control architecture for both macrocells and femtocells, based on Ji and Huang's study [10]. They treat macrocell users as game players and thus restrict the RRM of macrocells to the utility function and actions specified by the game. The scheme proposed by Jo et al. [7] adjusts the transmit power of femtocell users proportional to the fed-back interference level of macrocells and does not require any change of the macrocell RRM. However, they focused on protecting the macrocell's UL only and did not provide any convergence analysis. Moreover, both did not consider the feedback delay that affects the convergence of an algorithm. Yavuz et al. [4] proposed an attenuator-adjustment scheme in which a femtocell user under high UL interference is given room for increasing its transmit power due to the increased attenuation. This scheme is restricted to femtocell UL protection.

Recently, there have been a few proposals targeting OFDMA systems. A comparative study of orthogonal and co-channel deployments was reported in [11], showing that the optimal strategy depends on traffic load and resource split between macro- and femto-cells. Sundaresan and Rangarajan [12] proposed two models of femtocell management: isolated and coupled. In the isolated model, a macro BS and femto BSs in close proximity are allocated orthogonal timefrequency resources while the coupled model partly relaxes such a constraint for achieving higher total utility. However, they require strict time synchronization between macro and femto BSs and may incur high signaling overhead since two-way signaling from both macro and femto BSs to the network controller is invoked on each population change. The coupled model premises that femto BSs overhear the resource scheduling information of macrocell users, for which femto BSs need to be equipped with an extra receiving module with self-interference cancelation capability (difficulties and some solutions of the over-the-air feedback will be discussed in Section II.B). Moreover, both models impose some degree of design restrictions on the RRM of macro BSs for dynamic adjustment of resource split. Jin and $\mathrm{Li}$ [13] proposed a cognitive WiMax femtocell architecture exploiting cognitive sensing and multi-hop transmission among macrocell users which require substantial changes to user devices as well as BSs.

There have also been proposals for use of methods other than radio resource scheduling. Lopez-Perez et al. [14] proposed an intracell handover-based approach. Their main idea is that when a macrocell user suffers from a nearby femtocell's interference, the serving macrocell hands the user over to a less-interfered frequency channel, or evicts all interfering femtocells to other frequency channels. This approach can be interpreted as dynamic frequency assignment to macroand femto-cells. There have been other proposals focusing on optimal frequency assignment to overlaid cells. A decentralized frequency assignment strategy for femtocell networks was proposed by Chandrasekhar and Andrews [15], which depends on per-tier throughput, loading and QoS requirement, accounting for co-channel interference. Frequency partitioning and assignment approaches proposed for macrocell-microcell systems [16] [17] can be also extended to femtocell networks. However, these approaches, when used in a dynamic manner, incur service interruptions to ongoing connections. Moreover, they cannot handle the situation where every frequency channel is completely populated with femtocells.

\section{Contributions}

To overcome the above limitations of existing schemes, we propose a new, distributed and self-organizing femtocell management architecture for UL communications, called Complementary TRi-control Loops (CTRL), which is applicable to, but not restricted to, OFDMA-based cellular systems. The key idea behind CTRL is the multiple control loops optimally designed with different objectives while complementing each other toward a common goal. The control loops and their complementary interactions are summarized as follows. The maximum transmit power control (MTXPC) loop determines the maximum transmit power of femtocell users based on the fed-back macrocell UL load margin for protection of the macrocell UL communications. One distinct feature of the MTXPC loop is that the global femtocell status is estimated without extra signaling, and the control parameters are selftuned accordingly for stability, enabling a distributed and selforganizing operation. The target SINR control (TSINRC) loop enables utility-optimal resource coordination among femtocells without signaling between them. We show that a noncooperative optimal resource-allocation game among femtocells reaches a Nash equilibrium. The instantaneous transmit power control (ITXPC) loop controls the transmit power of a femtocell user such that the target SINR determined by the TSINRC loop is achieved on a small time-scale (e.g., frame) against bursty interference from other nearby macrocell and femtocell users, thus protecting the femtocell's UL.

CTRL meets all the requirements mentioned earlier. That is, CTRL does not require modification of the RRM of macro BSs, thus enabling smooth and non-disruptive migration of cochannel femtocells into existing cellular networks. In addition, the control algorithms of CTRL achieve convergence under the provided conditions in the presence of time-varying and unpredictable environmental changes, such as user traffic, other cells' interferences, threshold value, etc. Besides, CTRL is compatible with legacy user devices since it does not impose any non-standard operation on them. CTRL is software-based without increasing the hardware cost and can be improved further with an extra receiver module enabling the over-theair feedback (as detailed in Section II).

The rest of this paper is organized as follows. Section II describes the system model. Section III presents the CTRL architecture, while Section IV specifies the control algorithms. Section V evaluates the architecture via simulation and Section VI concludes the paper.

\section{SySTEM MOdEL}

This section describes the network architecture under consideration and two implementation alternatives for macrocell feedback. 


\section{A. Network Architecture}

In a typical two-tier femtocell network architecture, femtocells are overlaid on macrocells. The set of macrocells $\mathcal{M}=\{1, \ldots, M\}$ and the set of femtocells $\mathcal{F}=\{1, \ldots, F\}$ use a pre-specified frequency band. The frequency band is composed of multiple resource blocks for OFDMA, each of which is a group of contiguous subcarriers and also the minimum scheduling granularity. For example, in 3GPP LongTerm Evolution (LTE), 12 contiguous subcarriers compose a resource block ( 5 resource blocks in $900 \mathrm{kHz}$ band) [18]. Cell $m$ operates under the control of BS $m$. The set of users served by femtocell $f \in \mathcal{F}$ is denoted as $\mathcal{F}_{u}^{f}=\left\{f_{1}, \ldots, f_{\left|\mathcal{F}_{u}^{f}\right|}\right\}$ where $f_{l}$ indicates the $l$-th user (or its index) served by femtocell $f$ and $\left|\mathcal{F}_{u}^{f}\right|$ is the cardinality of the set $\mathcal{F}_{u}^{f}$. Each femtocell user is uniquely identified with a positive-integer index as follows. The user set of femtocell 1 is denoted as $\mathcal{F}_{u}^{1}=\left\{1, \ldots,\left|\mathcal{F}_{u}^{1}\right|\right\}$, that of femtocell 2 as $\mathcal{F}_{u}^{2}=\left\{\left|\mathcal{F}_{u}^{1}\right|+1, \ldots,\left|\mathcal{F}_{u}^{1}\right|+\left|\mathcal{F}_{u}^{2}\right|\right\}$, and so on. This indexing rule is generalized as $f_{l}=\sum_{f^{\prime}<f}\left|\mathcal{F}_{u}^{f^{\prime}}\right|+l$ where $1 \leq l \leq\left|\mathcal{F}_{u}^{f}\right|$. Let $\mathcal{F}_{u}=\bigcup_{f \in \mathcal{F}} \mathcal{F}_{u}^{f}$. Macro and femto BSs are assumed to use an identical OFDMA technology (note that CTRL is also applicable to different and even heterogenous access technologies with a straightforward extension).

\section{B. Macrocell Load Feedback}

To protect macrocell's UL, femto BSs need to know the current status of macrocells, as assumed in [6] [7], which may be enabled by feedback from macro BSs, called macrocell feedback. We define the cell load as a conceptual metric which measures the degree of the crowdedness of a wireless channel. ${ }^{3}$ We then assume that a macro BS feeds back its cell load margin defined as the difference between the current cell load and a load threshold; the cell load margin is positive when the current load is lower than the threshold; otherwise, it is negative. The cell load margin is derived for each resource block. ${ }^{4}$ Next, we describe two implementation alternatives for macrocell feedback, which differ in delay and cost.

1) Feedback over wired networks: First, we adopt the approach that femto BSs receive macrocell feedback via wired networks. We can realize this by (1) defining a direct signaling interface between macro- and femto-cells or (2) using an existing signaling interface between BSs and an operation, administration and management (OAM) server residing in the operator's core network. The first method is attractive in that it works without a central OAM server, but requires costly standardization since no signaling interface has been defined between macro and femto BSs in current standards. In contrast, signaling interfaces for OAM are generally vendorspecific, and thus, the second method can be readily applicable. The second method can be implemented as follows. We assume that, as in general cellular networks, every BS has a logical connection to an OAM server which BSs receive initial configuration settings from and occasionally report their status to. The OAM server dedicated to femtocells is called the femtocell manager. Each macro BS periodically reports its cell load margin to the OAM server. Then, the

\footnotetext{
${ }^{3}$ Our definition of cell load may not be consistent with generally-used ones.

${ }^{4} \mathrm{~A}$ larger granularity for deriving the cell load margin can be used to reduce signaling overhead at the expense of degraded control granularity.
}

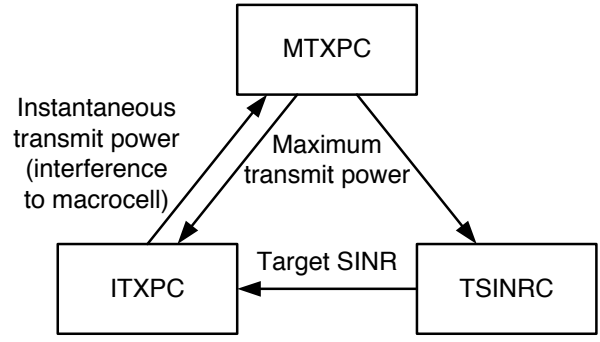

Fig. 2. Complementary interactions between control loops

macrocell OAM server forwards it to the femtocell manager. Finally, the femtocell manager sends it to the femto BSs subscribing to the feedback of the macro BS. To receive the feedback from proper macro BSs, femto BSs need to execute a subscription procedure; when powered on, a femto BS scans neighbor macrocells and reports the macrocell list of feedback subscriptions to the femtocell manager.

Feedback via wired networks does not require additional hardware of femto BSs, but incurs a larger delay than the other approach. We assume that this feedback delay is known to femto BSs. A rich body of research has tackled the delay estimation problem in the Internet (e.g., [19] [20]) and the environment under consideration is covered well by the cases explored in the literature. So, we omit the implementation detail of delay estimation.

2) Feedback over the air: In the second approach, femto BSs receive macrocell feedback directly from macro BSs over the air. Specifically, macro BSs broadcast the load margin information which is then overheard by femto BSs. This approach requires two issues to be resolved, implementation feasibility and standard violation, which are discussed next.

In the case of frequency-division duplex (FDD), femto BSs need to overhear macrocell signals at a frequency other than their original receiving frequency, i.e., they require an extra receiver module. The time-division duplex (TDD) case may also require this to enable full duplexing (receiving macrocell signals during an active transmission). On the other hand, if macro BSs feed back information at the same frequency that femto BSs use to transmit, femto BSs may not be able to demodulate macrocell signals due to significant selfinterference. This problem can be addressed by (1) macrocell's feedback at a frequency other than the femtocell's transmission frequency or (2) interference cancelation as in wireless relays [21]. The first solution is applicable when macro BSs use multiple frequency bands ${ }^{5}$ while the latter increases the cost further.

In order to broadcast the load margin information, modification of the legacy system information (SI) format [22] is required. In general, operators do not use every SI field in the standards. Thus, some unused SI fields can be redefined for the inclusion of feedback.

The delay of feedback over the air is the air propagation delay from a macro BS to femto BSs which is negligibly small.

\footnotetext{
${ }^{5}$ This is the typical case for single-carrier systems in urban areas due to high traffic demands.
} 


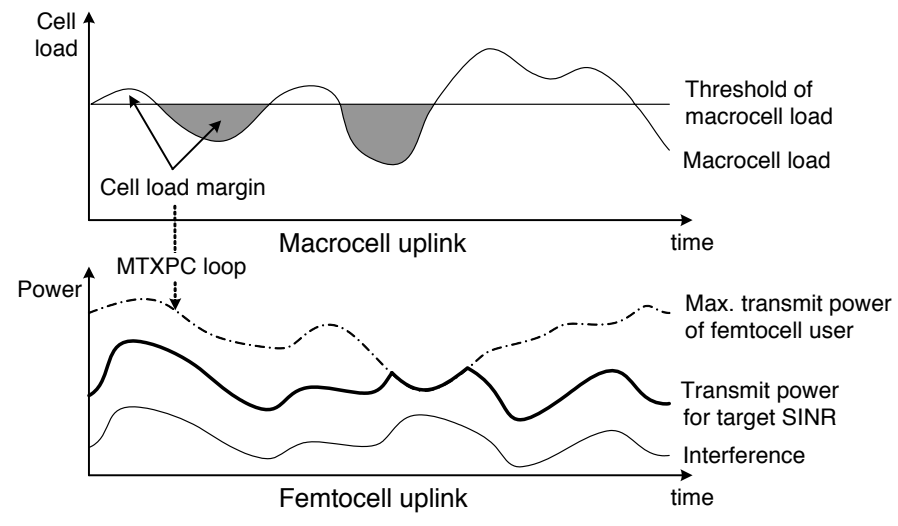

Fig. 3. Overview of the CTRL concept

\section{THE CTRL ARCHITECTURE}

This section outlines the architecture of CTRL. First, we describe the basic concept and design rationale of CTRL. Then, under the CTRL architecture, we formulate problems for the design of control algorithms.

\section{A. Overview of CTRL}

The goal of CTRL is to simultaneously achieve all the objectives listed in Section I.A (with the highest priority on macrocell UL protection) while meeting the requirements discussed in Section I.B. Each of the objectives can be considered as a subproblem of the femtocell interference problem. CTRL solves these subproblems individually using different control loops, constrains one's result by the others' according to the relationship depicted in Fig. 2 and finally produces a coordinated result. All decisions of the three control loops for a user are made by the femto BS he receives service from, based on the specified interaction rule between them. In what follows, we describe each of the control loops and complementary interactions thereof.

1) Maximum Transmit Power Control (MTXPC) Loop: The MTXPC loop is in charge of protecting macrocell's UL by controlling the maximum transmit power of femtocell users based on the fed-back macrocell load margin. As the cell load margin is defined for each resource block (as explained in Section II.B), an individual process of the MTXPC loop is required for each resource block. A positive macrocell load margin indicates that the macrocell has room for additional load, while a negative margin indicates an overloaded cell (Fig. 3). Assuming that the UL load of a cell is a monotonically-increasing function of the total received power, controlling the transmit power of femtocell users keeps, on average, macrocell's UL load below a threshold (the threshold is assumed to be higher than the macrocell's UL load without femtocell interference). Macrocell users' UL performance will, therefore, not be degraded below a specific level. An important feature of the MTXPC loop is that it controls the maximum transmit power, not the femtocell users' transmit power. Such an approach enables the other control loops to perform further optimization of femtocells based on their local condition.

The UL load of a macrocell originates from the three components: inner-macrocell user traffic, other macrocells' interference and femtocell interference. Prioritizing macrocell users, the maximum load that femtocells are allowed to contribute to is obtained by subtracting the inner and other macrocell load from the load threshold. However, a macro BS cannot distinguish other macrocells' interference from the femtocell's interference, and other macrocells' interference is uncontrollable. So, it cannot allocate an exact load portion to femtocells. Instead, a macro BS just provides its current (time-varying) load margin. Based on the margin, femtocells should then adapt their resource usage to the unknown portion they are allowed to use. We tackle this difficulty by modeling the unpredictable interference from other macrocells as a disturbance.

2) Target Signal to Interference and Noise Ratio Control (TSINRC) Loop: The TSINRC loop enables efficient resource (both resource blocks and power) coordination among neighboring femtocells based on local information, such as user-specific UL interference, transmission activity, channel condition, etc., to mitigate femtocell-to-femtocell interference. The coordination is achieved without signaling between femto BSs since no inter-femto BS signaling interface has been defined in standards. ${ }^{6}$ Therefore, femto BSs need to infer the current situation based on implicit feedback, such as the interference level and the achieved SINR. Finally, the result is conditioned on the maximum transmit power constraint obtained by the MTXPC loop.

3) Instantaneous Transmit Power Control (ITXPC) Loop: Although the TSINRC loop determines the target SINR, the short-term achievable SINR could fluctuate due to bursty interference (as mentioned in Section I.A) resulting in inconsistent user service quality. The interference sources are nearby macrocell users or other femtocell users. The ITXPC loop controls the instantaneous transmit power of a femtocell user on a small time-scale (e.g., frame) taking into account the user's local conditions such that the target SINR determined by the TSINRC loop is achieved in a short-term sense, as shown in Fig. 3. If the transmit power is simply set to the target SINR multiplied by the current interference, an abrupt change of interference due to transmission on/off of a nearby wireless source will lead to a drastic change of the transmit power and the interference to both macro and femto cells. Thus, the ITXPC algorithm should be designed to converge with neither overshoot nor oscillation. However, there exist certain local conditions which are uncontrollable or not accurately measurable, such as the interference from macrocell users, channel measurement error, etc. We model them as disturbances, and ITXPC is designed to asymptotically filter them out. Finally, the transmit power of femtocell users determined by the corresponding ITXPC loops collectively forms the femtocell interference to a macro BS and, consequently, influence the MTXPC loop. The result of the ITXPC loop is also conditioned on the maximum transmit power constraint obtained by the MTXPC loop.

${ }^{6}$ In LTE release 9, the inter-BS interface, named X2, is defined only between macro BSs. The interface between macro and femto BSs is under discussion for LTE release 10 . 


\section{B. Problem Formulation for Control Algorithms}

Throughout the paper, the "transmit power" is the power a user to transmit his data. The amount of the radiated energy per unit of time is thus obtained by multiplying a transmission activity factor to the transmit power. Let $p_{i, j}$ and $P_{i, j}$ denote the transmit power and the maximum transmit power of femtocell user $i$ at resource block $j$, respectively. The corresponding transmission activity factor, denoted by $a_{i, j}\left(0 \leq a_{i, j} \leq 1\right)$, is the ratio of the slots in which user $i$ transmits at resource block $j$, to the entire slots during a certain period, and determined by the traffic arrival pattern and resource scheduling of the user. The channel gain from user $i$ to BS $m$ at resource block $j$ is denoted by $h_{i, m}^{j}$. Let $\mathcal{J}=\{1, \cdots, J\}$ be the set of given resource blocks and $b_{i, j} \in\{0,1\}$ be the allocation indicator of user $i$ to resource block $j$. Here, $a_{i, j}$ and $b_{i, j}$ differ in that $b_{i, j}$ is determined by a femtocell scheduler on a per-slot basis while $a_{i, j}$ describes the activity status averaged over a longer period. ${ }^{7} \mathrm{We}$ also define the power allocation vectors: $\mathbf{p}_{i} \triangleq\left[p_{i, 1}, \cdots, p_{i, J}\right]^{\mathrm{T}}$ and $\mathbf{P}_{i} \triangleq\left[P_{i, 1}, \cdots, P_{i, J}\right]^{\mathrm{T}}$ for $i \in \mathcal{F}_{u} ; \mathbf{p}^{f} \triangleq\left[\mathbf{p}_{f_{1}}, \cdots, \mathbf{p}_{\left.f_{\mid \mathcal{F}_{u}^{f}}\right]^{\mathrm{T}}}\right.$ and $\mathbf{P}^{f} \triangleq\left[\mathbf{P}_{f_{1}}, \cdots, \mathbf{P}_{f_{\left|\mathcal{F}_{u}^{f}\right|}}\right]^{\mathrm{T}}$ for $f \in \mathcal{F} ; \mathbf{p} \triangleq\left[\mathbf{p}^{1}, \cdots, \mathbf{p}^{F}\right]^{\mathrm{T}}$ and $\mathbf{P} \triangleq\left[\mathbf{P}^{1}, \cdots, \mathbf{P}^{F}\right]^{\mathrm{T}} ; \mathbf{b}_{i} \triangleq\left[b_{i, 1}, \cdots, b_{i, J}\right]^{\mathrm{T}}$ for $i \in \mathcal{F}_{u}$ and $\mathbf{b}^{f} \triangleq\left[\mathbf{b}_{f_{1}}, \cdots, \mathbf{b}_{f_{\mid \mathcal{F}^{f}}}\right]^{\mathrm{T}}$ for $f \in \mathcal{F}$. Let $\gamma_{i, j}$ be user $i$ 's achieved SINR at resource block $j$ and the corresponding vectors be $\gamma_{i} \triangleq\left[\gamma_{i, 1}, \cdots, \gamma_{i, J}\right]^{\mathrm{T}}$ and $\gamma^{f} \triangleq\left[\gamma_{f_{1}}, \cdots, \gamma_{f_{\left|\mathcal{F}_{u}^{f}\right|}}\right]^{\mathrm{T}}$. Then, the control algorithms aim to find the above vectors that meet their objectives. The solution vectors yielded by the algorithms are denoted as $\mathbf{p}^{*}, \mathbf{P}^{*}, \mathbf{b}^{f *}$ and $\gamma^{f *}$ (target SINR), respectively.

Let the problems of the control loops be denoted by $\mathrm{Prob}_{1}$ (MTXPC), $\mathrm{Prob}_{2}$ (TSINRC), and $\mathrm{Prob}_{3}$ (ITXPC). Let $L_{t h, j}^{m}(t)$ and $L_{j}^{m}(t)$ be the load threshold and load of macrocell $m$ at time $t$, respectively, in resource block $j$. We define $e_{j}^{m} \triangleq L_{t h, j}^{m}-L_{j}^{m}$ as the load margin of macrocell $m$. Then, the basic objective of $\operatorname{Prob}_{1}$ is to let $\left[e_{j}^{m}(t)\right]^{+}$converge to zero. ${ }^{8}$ If there are enough load demands from femtocells to enable maximum spatial reuse within femtocells, the objective is to let $\left|e_{j}^{m}(t)\right|$ converge to zero. Thus, $\operatorname{Prob}_{1}$ is formulated as

$$
\operatorname{Prob}_{1}: \min _{\mathbf{P}} \lim _{t \rightarrow \infty}\left|e_{j}^{m}(t)\right| \text { for } m \in \mathcal{M}, j \in \mathcal{J} \text {. }
$$

$L_{j}^{m}$ consists of macrocell user portion $L_{M, j}^{m}$ and femtocell user portion $L_{F, j}^{m}$ such that $L_{j}^{m}(t)=L_{M, j}^{m}(t)+L_{F, j}^{m}(t)$ and $L_{F, j}^{m}(t)=\Gamma_{m}\left(I_{F, j}^{m}(\mathbf{p})\right)$ where $I_{F, j}^{m}(\mathbf{p})=\sum_{i} a_{i, j} h_{i, m}^{j} p_{i, j}$ and $\Gamma_{m}: \mathbb{R} \rightarrow \mathbb{R}$ is an interference-to-load function which is monotonically increasing in $I_{F, j}^{m}$. As mentioned earlier, the MTXPC loop controls $\mathbf{P}$ although $L_{F, j}^{m}$ is a function of $\mathbf{p}$. The only connection between them is $\mathbf{p} \preceq \mathbf{P}$. ${ }^{9}$ Under this limiting approach, the solution of $\mathrm{Prob}_{1}$ exists as shown below.

Proposition 1: If there exists feasible $\mathbf{p}^{*}$ such that $e_{j}^{m}=0$, so does $\mathbf{P}^{*}$.

\footnotetext{
${ }^{7}$ Suppose that $b_{i, j}(t)=0$ when a transmission buffer is empty in slot $t$. Then, one way to calculate $a_{i, j}$ is $a_{i, j}(k)=\sum_{t=k T}^{(k+1) T-1} b_{i, j}(t) / T$ where $k$ is a feedback interval index and $T$ is the duration of a feedback interval in number of slots.

${ }^{8}[x]^{+}=\max \{x, 0\}$.

${ }^{9}$ The curled inequality symbol $\succeq$ (and its strict form $\succ$ ) represents component-wise inequality.
}

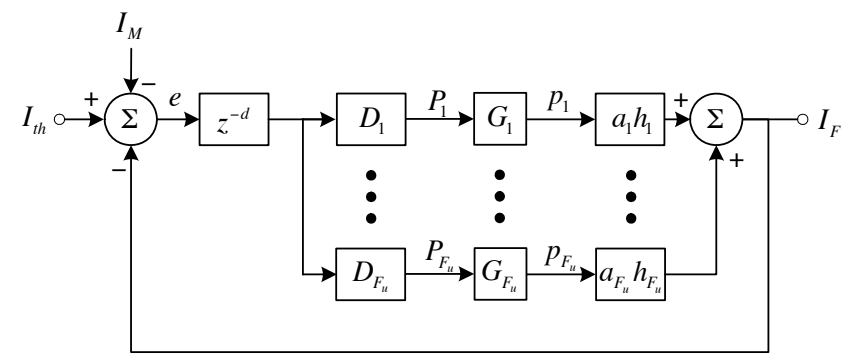

(a) MTXPC loop

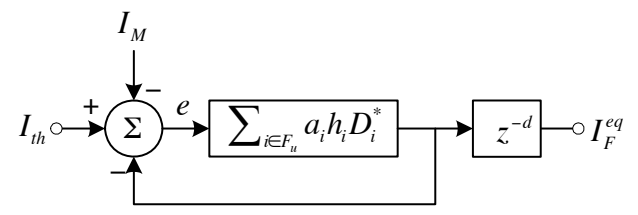

(b) Equivalent control loop

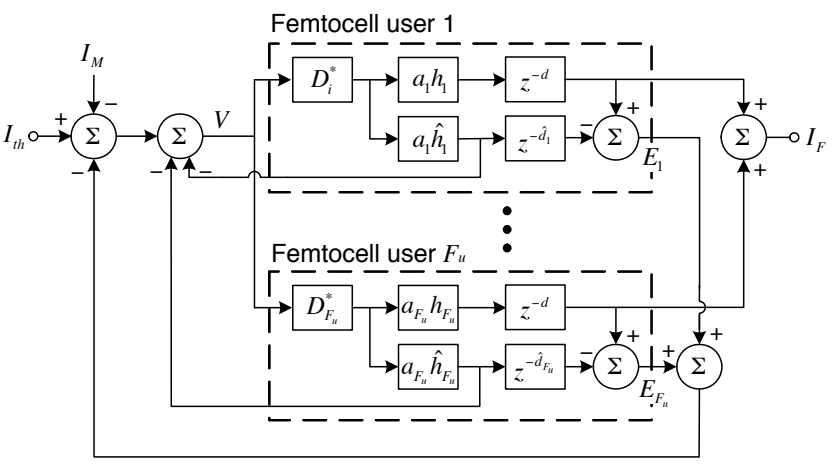

(c) MTXPC loop redrawn

Fig. 4. MTXPC loop diagram

Proof: Since $I_{F, j}^{m}(\mathbf{p}) \leq I_{F, j}^{m}(\mathbf{P})$ and $\Gamma_{m}$ is a monotonically increasing function, $\Gamma_{m}\left(I_{F, j}^{m}(\mathbf{p})\right) \leq \Gamma_{m}\left(I_{F, j}^{m}(\mathbf{P})\right)$. Suppose $0 \leq \Gamma_{m}<\infty$. Then, there always exists $\epsilon \geq 0$ such that $\Gamma_{m}\left(I_{F, j}^{m}(\mathbf{P})\right)=\Gamma_{m}\left(I_{F, j}^{m}\left(\mathbf{p}^{*}\right)\right)+\epsilon . I_{F, j}^{m}(\mathbf{P})=$ $\Gamma_{m}^{-1}\left(\Gamma_{m}\left(I_{F, j}^{m}\left(\mathbf{p}^{*}\right)\right)+\epsilon\right)$ and, due to the monotonicity of $\Gamma_{m}$, there also exists $\epsilon^{\prime} \geq 0$ such that $I_{F, j}^{m}(\mathbf{P})=$ $\Gamma_{m}^{-1}\left(\Gamma_{m}\left(I_{F, j}^{m}\left(\mathbf{p}^{*}\right)\right)\right)+\epsilon^{\prime}=I_{F, j}^{m}\left(\mathbf{p}^{*}\right)+\epsilon^{\prime}$. Here, $I_{F, j}^{m}(\mathbf{P})=$ $I_{F, j}^{m}\left(\mathbf{p}^{*}\right)+\epsilon^{\prime} \geq 0$ and thus it forms an affine hyperplane in an $\left|\mathcal{F}_{u}\right|$-dimensional Euclidean space of $\mathbf{P}$ lower-bounded by $\mathbf{p}^{*}$, which ensures the existence of $\mathbf{P}^{*}$ ( $\epsilon$ is determined by the other two control loops).

We formulate $\mathrm{Prob}_{2}$ as a non-cooperative $N$-person game that each femtocell maximizes his utility function without signaling with the others, while being conditioned on the result of $\operatorname{Prob}_{1}$, i.e., $\mathbf{P}^{*}$ :

$$
\operatorname{Prob}_{2}: \max _{\left(\mathbf{p}^{f}, \mathbf{b}^{f}, \boldsymbol{\gamma}^{f}\right) \in \mathcal{U}_{f}} u_{f}\left(\mathbf{p}^{f}, \mathbf{b}^{f}, \gamma^{f}\right) \text { for } f \in \mathcal{F}
$$

where $u_{f}$ is the utility function of femtocell $f$ and $\mathcal{U}_{f}$ is the feasible region (described in Section IV.B). Finally, $\operatorname{Prob}_{3}$ is to let $e_{i, j} \triangleq \gamma_{i, j}^{*}-\gamma_{i, j}$ converge to zero for $i \in \mathcal{F}_{u}, j \in \mathcal{J}$ where $\gamma_{i, j}^{*}$ is the result of $\operatorname{Prob}_{2}$. Thus, $\operatorname{Prob}_{3}$ is expressed, similarly to $\operatorname{Prob}_{1}$, as

$$
\operatorname{Prob}_{3}: \min _{p_{i, j} \leq P_{i, j}^{*}} \lim _{t \rightarrow \infty}\left|e_{i, j}(t)\right| \text { for } i \in \mathcal{F}_{u}, j \in \mathcal{J} \text {. }
$$




\section{Control Algorithms}

We now design control algorithms to solve the problems formulated in the previous section.

\section{A. MTXPC Algorithm}

Prob $_{1}$ can be considered as a steady-state tracking problem from a control-theoretic point of view, i.e., a control effort lets a macrocell's load track the specified threshold value. Here, $e_{j}^{m}$ is interpreted as the tracking error.

For details of the algorithm, we consider rise over thermal (RoT) as a cell load metric, i.e., $L_{j}^{m}=\left(I_{j}^{m}+\sigma_{j}^{2}\right) / \sigma_{j}^{2}$, where $I_{j}^{m}$ is the total power received at macro BS $m$ and $\sigma_{j}^{2}$ is the thermal noise within resource block $j$. Then, $e_{j}^{m}=\left(I_{t h, j}^{m}-I_{j}^{m}\right) / \sigma_{j}^{2}$. Without loss of generality, we can simply let $e_{j}^{m}=I_{t h, j}^{m}-I_{j}^{m}$. We denote the power received at macro BS $m$ from macrocell and femtocell users by $I_{M, j}^{m}$ and $I_{F, j}^{m}$, respectively, so that $I_{j}^{m}=I_{M, j}^{m}+I_{F, j}^{m}$. For brevity of presentation, we drop the superscript and subscript $m$. We also drop the resource block index $j$ in this subsection since each MTXPC loop process per resource block follows an identical work flow.

Let $T$ be the length of the macrocell feedback interval. Then, the MTXPC loop can be modeled as a discrete-time system whose state changes at an interval boundary. Let $x(k)$ denote variable $x$ during the $k$-th interval, i.e., $[k T,(k+1) T)$. Then, $e(k)$ is written as

$$
e(k)=I_{t h}(k)-I(k)=I_{t h}(k)-I_{M}(k)-I_{F}(k) .
$$

The MTXPC loop can be represented as a closed-loop control system depicted in Fig. 4(a). The chain reactions shown in the figure can be explained as follows. A macro BS sends $e$ to the femto BSs subscribing to its feedback. Suppose that the femto BSs receive $e$ with the feedback delay $d \times T$ (represented as $z^{-d}$ in the figure). Upon reception of $e$, the femto BSs update $P_{i}$ of their users $i \in \mathcal{F}_{u}$ with the user-specific controller $D_{i}$, i.e., $P_{i}(k)=D_{i}(e(k-d))$. Then, $p_{i}=G_{i}\left(P_{i}\right)=\min \left\{\widetilde{p}_{i}, P_{i}\right\}$ where $\widetilde{p}_{i}$ is determined by the other two control loops. We can thus let $p_{i}(k)=P_{i}(k)-\epsilon_{i}(k)$ where $0 \leq \epsilon_{i}(k) \leq P_{i}(k) . \epsilon_{i}(k)$ is time-varying based on user $i$ 's local condition. Finally, $I_{F}$ is updated as:

$$
\begin{aligned}
I_{F}(k) & =\sum_{i \in \mathcal{F}_{u}} a_{i} h_{i} p_{i}(k)=\sum_{i \in \mathcal{F}_{u}} a_{i} h_{i}\left(P_{i}(k)-\epsilon_{i}(k)\right) \\
& =\sum_{i \in \mathcal{F}_{u}} a_{i} h_{i} D_{i}(e(k-d))-\epsilon(k)
\end{aligned}
$$

where $h_{i}$ is the channel gain from femtocell user $i$ to a tagged macro BS and $\epsilon \triangleq \sum_{i \in \mathcal{F}_{u}} a_{i} h_{i} \epsilon_{i}$. The $z$-transform plays an important role in the analysis of discrete-time systems described by linear difference equations like Eq. (5). It converts the manipulation of difference equations to purely algebraic problems, thus enabling us to obtain the inputoutput relationship of a system as a transfer function that facilitates the understanding of system behavior. We denote the $z$-transform of $x(k)$ by simply $x(z)$. Applying the $z$-transform to Eqs. (4) and (5), and combining the results, we have

$$
\begin{aligned}
I_{F}(z)= & \frac{z^{-d} \sum_{i \in \mathcal{F}_{u}} a_{i} h_{i} D_{i}(z)}{1+z^{-d} \sum_{i \in \mathcal{F}_{y}} a_{i} h_{i} D_{i}(z)}\left(I_{t h}(z)-I_{M}(z)\right) \\
& +\frac{1}{1+z^{-d} \sum_{i \in \mathcal{F}_{u}} a_{i} h_{i} D_{i}(z)} \epsilon(z) .
\end{aligned}
$$

1) Decoupling of the Feedback Delay Component: The control system in Fig. 4(a) is difficult to analyze since it contains the delay component $z^{-d}$ within the feedback loop [23]. Thus, we consider an equivalent system in Fig. 4(b) where the delay component is outside the feedback loop. $I_{F}$ of this system is referred to as $I_{F}^{e q}$. Here, for ease of design, we temporarily ignore $\epsilon$. Later, we prove in Proposition 3 that the resultant system works as desired even with a nonzero $\epsilon$. Using a procedure similar to Eq. (6),

$$
I_{F}^{e q}(z)=\frac{\sum_{i \in \mathcal{F}_{u}} a_{i} h_{i} D_{i}^{*}(z)}{1+\sum_{i \in \mathcal{F}_{u}} a_{i} h_{i} D_{i}^{*}(z)} z^{-d}\left(I_{t h}(z)-I_{M}(z)\right)
$$

and, by equating $I_{F}(z)$ and $I_{F}^{e q}(z)$, we have

$$
\sum_{i \in \mathcal{F}_{u}} a_{i} h_{i} D_{i}(z)=\frac{\sum_{i \in \mathcal{F}_{u}} a_{i} h_{i} D_{i}^{*}(z)}{1+\left(1-z^{-d}\right) \sum_{i \in \mathcal{F}_{u}} a_{i} h_{i} D_{i}^{*}(z)} .
$$

Then, we define $D_{i}(z)$ as

$$
D_{i}(z)=\frac{D_{i}^{*}(z)}{1+\left(1-z^{-d}\right) \sum_{i \in \mathcal{F}_{u}} a_{i} h_{i} D_{i}^{*}(z)}
$$

which satisfies Eq. (8). That is, if we use the above $D_{i}(z)$, the system becomes equivalent to that of Fig. 4(b). This type of controller is called Smith predictor [23] which is known to offer better response than classical (PID or PI) controllers if there exists a time lag within a control loop [24]. Conceptually, the Smith predictor feeds back a simulated system output to cancel the true system output so as to alleviate the effect of a pure time delay. More on the feedback structure with the Smith predictor will be discussed in the next paragraph. Note that $D_{i}(z)$ is a controller implemented in a femto BS, and thus $d$ and $h_{i}$ in Eq. (9) are in practice the estimated values. The estimation error of $d$ may come from network congestion dynamics and that of $h_{i}$ may result from channel non-reciprocity in FDD, user mobility, etc. So, we let $d \rightarrow \hat{d}$ and $h_{i} \rightarrow \hat{h_{i}}$ in Eq. (9) to distinguish them from the true ones.

For better understanding of the resultant system, we rewrite the system transfer function by applying Eq. (8) to Eq. (6) as

$$
I_{F}(z)=\frac{\sum_{i \in \mathcal{F}_{u}} D_{i}^{*}(z) z^{-d} a_{i} h_{i}\left(I_{t h}(z)-I_{M}(z)\right)}{1+\sum_{i \in \mathcal{F}_{u}} D_{i}^{*}(z) a_{i} \hat{h}_{i}+\sum_{i \in \mathcal{F}_{u}} D_{i}^{*}(z)\left(z^{-d} a_{i} h_{i}-z^{-d} a_{i} h_{i}\right)} .
$$

Define $V$ as the control input to $D_{i}^{*}$. Then,

$$
I_{F}(z)=\sum_{i \in \mathcal{F}_{u}} V(z) D_{i}^{*}(z) z^{-d} a_{i} h_{i} .
$$

Let $E_{i}(z) \triangleq V(z) D_{i}^{*}(z)\left(z^{-d} a_{i} h_{i}-z^{-\hat{d}} a_{i} \hat{h}_{i}\right) . E_{i}(z)$ can be interpreted as the output discrepancy of user $i$ resulting from errors in estimating $d$ and $h_{i}$. Then, equating Eqs. (10) and (11), and after simplification, we get

$$
V(z)=I_{t h}(z)-I_{M}(z)-\sum_{i \in \mathcal{F}_{u}}\left(V(z) D_{i}^{*}(z) a_{i} \hat{h}_{i}+E_{i}(z)\right) .
$$

Based on Eqs. (11) and (12), we can redraw the system as in Fig. 4(c). In the redrawn system, each user has two 
estimation error-related feedback lines with and without a delay component. This dual feedback structure provides a certain level of robustness to the errors in estimating $d$ and $h_{i}$. The simulation results in Section $\mathrm{V}$ show that $e$ is bounded even for up to $100 \%$ overestimation of $d$ and $h_{i}$. With accurate estimation of $d$ and $h_{i}$, i.e., $\hat{d}=d$ and $\hat{h}_{i}=h_{i}$, the delay component is completely compensated within a femto BS's controller $D_{i}$ and the redrawn system becomes equivalent to the system of Fig. 4(b).

2) Controller Design: We can now design $D_{i}^{*}$ without considering a delay component. To avoid a drastic change of $P_{i}$, we adopt an additive increase/decrease approach for controlling it as

$$
P_{i}(k+1)=P_{i}(k)+\Delta P_{i}(k)
$$

and $\Delta P_{i}$ is controlled using a controller $C_{i}$ based on $e$ : $\Delta P_{i}(k)=C_{i}(e(k))$. Suppose that $C_{i}$ is a linear controller. ${ }^{10}$ Then, $P_{i}$ is expressed in the $z$-domain as

$$
P_{i}(z)=D_{i}^{*}(z) e(z)=\frac{C_{i}(z)}{z-1} e(z) .
$$

Let $C_{i}(z) \triangleq q_{i} C(z)$ where $q_{i}$ is a user-specific constant determined based on the user priority and control policy, and $C(z)$ is a network-wide common femtocell controller. Then, $P_{i}(z)=q_{i} P(z)$ where $P(z)=\frac{C(z)}{z-1} e(z)$ is the variable identically calculated by all femtocell users subscribing to the same macrocell feedback. For simplicity of presentation, we define $Q \triangleq \sum_{i \in \mathcal{F}_{u}} a_{i} h_{i} q_{i}$ so that Eq. (5) is rewritten as $I_{F}=Q P-\epsilon$ in both time and $z$ domains.

3) Stability Analysis: As a special case, we consider a Proportional-Integral controller [25] for $C$ as:

$$
C(z)=K_{P}+K_{I}\left(1-z^{-1}\right)^{-1}
$$

where $K_{P}$ and $K_{I}$ are constants. ${ }^{11}$ For $C$, we explore stability and convergence to the optimal point under time-varying and unpredictable $I_{t h}, I_{M}$ and $\epsilon$.

Proposition 2: With accurate estimation of $d$ and $h_{i}$, the MTXPC loop is stable if and only if $0<K_{P}<2 / Q$ and $0<K_{I}<4 / Q-2 K_{P}$. (The proof is presented in Appendix.)

Proposition 3: The MTXPC loop converges to the optimal point, i.e., $e \rightarrow 0$, under unpredictable time-varying $I_{t h}, I_{M}$ and $\epsilon$. (The proof is presented in Appendix.)

Finally, from Eqs. (9),(14) and (15),

$$
\begin{aligned}
P_{i}(z) & =D_{i}(z) z^{-d} e(z) \\
& =\frac{q_{i}\left(\hat{Q}_{K} \hat{Q}^{-1}-K_{P} z^{-1}\right) \cdot z^{-d} e(z)}{z+\hat{Q}_{K}-2-\left(K_{P} \hat{Q}-1\right) z^{-1}-\hat{Q}_{K} z^{-\hat{d}}+K_{P} \hat{Q} z^{-\hat{d}-1}}
\end{aligned}
$$

\footnotetext{
${ }^{10} \mathrm{~A}$ controller is linear if its output is a linear function of past controller outputs and other system variables. All types of PID controllers can be viewed as special cases of linear controllers.

${ }^{11}$ The transient behavior of a PI controller is known to be generally more stable than a PID controller in the presence of noise. This is because the derivative action of a PID controller is sensitive to noise and causes jittery output. In the femtocell control system considered in this paper, there are several noise sources, such as other macrocells' interference, the gap between the maximum transmit power and the actual one, etc. Thus, a PI controller is more suitable for our case.
}

where $\hat{Q}=\sum_{i \in \mathcal{F}_{u}} a_{i} \hat{h}_{i} q_{i}$ and $\hat{Q}_{K}=\left(K_{P}+K_{I}\right) \hat{Q}$, and thus $P_{i}$ is obtained in the time domain as

$$
\begin{aligned}
P_{i}(k+1)= & {\left[2-\hat{Q}_{K}\right] P_{i}(k)+\left(K_{P} \hat{Q}-1\right) P_{i}(k-1) } \\
& +\hat{Q}_{K} P_{i}(k-\hat{d})-K_{P} \hat{Q} P_{i}(k-\hat{d}-1) \\
& +q_{i} \hat{Q}_{K} \hat{Q}^{-1} e(k-d)-q_{i} K_{P} e(k-d-1) .
\end{aligned}
$$

Note, however, that deriving $P_{i}$ requires the knowledge of $Q$ (or $\hat{Q}$ ) which corresponds to the global femtocell status composed of activity factors, channel gains and user-specific constants of other femtocell users. This requirement of global knowledge makes it difficult to realize a distributed and selforganizing operation. To resolve this issue, we propose a $Q$ estimation scheme which operates without extra signaling.

4) Self-Organizing MTXPC Loop: The proposed $Q$ estimation scheme is based on adaptive control theory [26] in the sense that the system parameter $Q$ is estimated from observed data and the control parameters are tuned accordingly. Within the adaptive control framework, we can consider $e$ as an observed variable and $P$ as the input variable. From Eqs. (4) and (5), $e(k)=-Q P(k)+v(k)$, which is called the true process, where $v(k)=I_{t h}(k)-I_{M}(k)+\epsilon(k)$ is the sum of disturbance terms. We design our process model as $e(k)=-\hat{Q}(k) P(k)$ and $\hat{Q}(k)$ indicates the estimated value of $Q$ at interval $k$. The process model does not include $v$ since we don't have accurate knowledge of how $v$ is generated, but we show in Proposition 4 that the estimation converges if $P$ or $v$ are nonzero. $\hat{Q}$ is updated every feedback interval reflecting the estimation error, denoted as $\varepsilon$, as described below:

$$
\begin{aligned}
& \hat{Q}(k)=\hat{Q}(k-1)+K(k) \varepsilon(k) \\
& \varepsilon(k)=e(k-d)+\hat{Q}(k-1) P(k-d)
\end{aligned}
$$

where $K$ is the weighting factor of correction. Here, $e(k-d)$ is used for the update at time $k$ since it is the latest observed variable due to the feedback delay. To determine $K$, we adopt the recursive least squares algorithm [26]:

$$
\begin{aligned}
& K(k)=R(k-1) P(k-d)\left(\lambda+R(k-1) P^{2}(k-d)\right)^{-1} \\
& R(k)=(1-K(k) P(k-d)) R(k-1) / \lambda
\end{aligned}
$$

where $\lambda$ is a forgetting factor for tracking time-varying $Q$ $(0<\lambda \leq 1)$.

Proposition 4: The least squares estimation of $Q$ converges to a unique value if and only if $P$ or $v$ are nonzero.

Proof: From Theorem 2.6 in [26], when the number of estimated parameters is $n$, the least squares estimation is convergent if the input signal is persistently exciting of order $n$, i.e., $\lim _{k^{\prime} \rightarrow \infty} \frac{1}{k^{\prime}} \sum_{k=1}^{k^{\prime}}(A(q) u(k))^{2}>0$ for all nonzero polynomials $A$ of degree $n-1$ or less and input signal $u$ where $q$ is the forward shift operator. In our case, $n=1$ and thus the input signal ( $P$ or $v$ ) should be persistently exciting at least of order one for convergence. Let $A(q)=a_{0}$ (degree zero). Then, $\frac{1}{k^{\prime}} \sum_{k=1}^{k^{\prime}}(A(q) u(k))^{2}=\frac{a_{0}^{2}}{k^{\prime}} \sum_{k=1}^{k^{\prime}}(u(k))^{2}>0$ if and only if $u$ is nonzero. Therefore, nonzero $P$ and $v$ are persistently exciting at least of order one, and this guarantees the convergence of the estimation.

Based on the estimated $\hat{Q}, K_{P}$ and $K_{I}$ are tuned such that they fall within the stability region obtained in Proposition 2. We use a simple tuning procedure: $K_{P}(k+1)=\alpha / \hat{Q}(k)$ and $K_{I}(k+1)=\beta / \hat{Q}(k)$ where $\alpha$ and $\beta$ are pre-configured 
parameters such that the controller is responsive enough while $K_{P}=\alpha / Q$ and $K_{I}=\beta / Q$ meet the stability region. We can set $\hat{Q}(0) \geq Q$ so that $K_{P}$ and $K_{I}$ are within the stability region from the beginning. Such an initialization of $\hat{Q}$ can be done with the aid of the femtocell manager since it continuously monitors all femtocells to some degree.

\section{B. TSINRC Algorithm}

The goal of the TSINRC algorithm is to let femtocell users reach a Nash equilibrium [27] in a fully-distributed manner by solving $\mathrm{Prob}_{2}$. In [28], the authors showed that a non-cooperative optimal power allocation game in OFDMA reaches a Nash equilibrium, provided subcarrier (or resource block) allocation has been pre-determined. We show that a non-cooperative optimal resource block and power allocation game also reaches a Nash equilibrium when $b_{i, j}$ is relaxed as a real variable in $[0,1]$. In this case, $b_{i, j}$ can be considered as the time-sharing factor for one resource block. ${ }^{12}$ We define the utility function of femtocell $f$ as

$$
\begin{aligned}
& u_{f}\left(\mathbf{p}^{f}, \mathbf{b}^{f}, \gamma^{f}\right) \\
& =\sum_{i \in \mathcal{F}_{u}^{f}} \sum_{j \in \mathcal{J}} b_{i, j} W \log _{2}\left(1+\gamma_{i, j} / \Omega\right)-\sum_{i \in \mathcal{F}_{u}^{f}} \sum_{j \in \mathcal{J}} \mu_{i, j} h_{i}^{j} b_{i, j} p_{i, j}
\end{aligned}
$$

which is an extended version of $\mathrm{Ji}$ and Huang's [10] to OFDMA systems. In the femtocell problem, the first and second terms of the right-hand side can be interpreted as a reward for capacity gain and a penalty for interference to macrocells, respectively, as pointed out by Chandrasekhar et al. [6]. $\mu_{i, j}$ is a balancing constant between these two terms; $W$ is the bandwidth of one resource block; and $\Omega=-\ln (5 \mathrm{BER}) / 1.6$ is a constant SNR gap for the required bit error rate (BER) [29]. Here, $p_{i, j}$ and $\gamma_{i, j}$ have the following relationship:

$$
\gamma_{i, j}=\frac{h_{i, S(i)}^{j} p_{i, j}}{I_{i, j}\left(\mathbf{p}_{-i}\right)}
$$

where $S(i)$ is the serving femto BS of user $i$; $\mathbf{p}_{-i}$ is the transmit power vector of all femtocell users except $i$ and $I_{i, j}\left(\mathbf{p}_{-i}\right)$ indicates the UL interference plus the thermal noise user $i$ experiences at resource block $j$, including the interference from macrocell users.

The feasible region of $\left(\mathbf{p}^{f}, \mathbf{b}^{f}\right)$, denoted by $\mathcal{U}_{f}$, is defined as $\left\{\left(\mathbf{p}^{f}, \mathbf{b}^{f}\right) \mid \mathbf{p}_{i} \in \mathbb{P}^{f}, 0 \preceq \mathbf{p}_{i} \preceq \mathbf{P}_{i}, \mathbf{b}^{f} \in \mathbb{B}^{f}, 0 \preceq\right.$ $\left.\mathbf{b}^{f} \preceq 1\right\}$ where $\mathbb{P}^{f}=\left\{\mathbf{p}_{i} \mid \sum_{j} \bar{b}_{i, j} p_{i, j} \leq P_{\max }\right\}$ and $\mathbb{B}^{f}=\left\{\mathbf{b}^{f} \mid \sum_{i} b_{i, j} \leq 1\right\} . \mathbb{P}^{f}$ and $\mathbb{B}^{f}$ follow from the transmit power limitation of a user device and the orthogonal resource allocation or time-sharing constraint among users within a cell, respectively.

Proposition 5: The non-cooperative resource block and power allocation game $\mathrm{Prob}_{2}$ has a Nash equilibrium if $\mu_{i, j}$ is large enough.

\footnotetext{
${ }^{12}$ Relaxation of $b_{i, j}$ to a real variable has been considered in the literature. Under this relaxation, $b_{i, j}$ can be interpreted as the fraction of time user $i$ occupies resource block $j$. For example, suppose that $b_{1, j}=0.6$ and $b_{2, j}=$ 0.4 . Then, two basic scheduling designs can be considered: (1) users 1 and 2 occupy resource block $j$ alternately; user 1 uses it for 3 consecutive slots and then user 2 for 2 slots; (2) a random number is generated in every slot; if the number is smaller than 0.6, user 1 uses the block; otherwise, user 2 uses it. Our simulation adopted the second one.
}

Proof: From [30], the solution of $\mathrm{Prob}_{2}$ is a Nash equilibrium if, for all $f \in \mathcal{F},(1) \mathcal{U}_{f}$ is a nonempty, convex, and compact subset of some Euclidean space $\mathbb{R}^{\sum_{f}\left|\mathcal{F}_{u}^{f}\right| \times J}$; (2) $u_{f}(\mathbf{p}, \mathbf{b}, \gamma)$ is continuous in $(\mathbf{p}, \mathbf{b})$ and quasi-concave in $\left(\mathbf{p}^{f}, \mathbf{b}^{f}\right)$. We show that $\operatorname{Prob}_{2}$ meets these two conditions if $\mu_{i, j}$ is within a provided range, as follows.

$\mathbb{P}^{f}$ is an epigraph of a convex function, which is convex, and $\left\{\mathbf{p}_{i} \mid 0 \preceq \mathbf{p}_{i} \preceq \mathbf{P}_{i}\right\}$ is also convex. Likewise, $\mathbb{B}^{f}$ (closed halfspace) and $\left\{\mathbf{b}^{f} \mid 0 \preceq \mathbf{b}^{f} \preceq 1\right\}$ are convex sets. Since convexity is preserved under set intersection, $\mathcal{U}_{f}$ is convex. $\mathcal{U}_{f}$ is closed and bounded, assuring its compactness.

It can be easily shown that $\frac{\partial^{2} u_{f}}{\partial p_{i, j} \partial p_{i^{\prime}, j^{\prime}}} \leq 0$ and $\frac{\partial^{2} u_{f}}{\partial b_{i, j} \partial b_{i^{\prime}, j^{\prime}}} \leq 0 . \frac{\partial^{2} u_{f}}{\partial p_{i, j} \partial b_{i^{\prime}, j^{\prime}}}=\frac{\partial^{2} u_{f}}{\partial b_{i, j} \partial p_{i^{\prime}, j^{\prime}}}=0$ if $(i, j) \neq$ $\left(i^{\prime}, j^{\prime}\right) \cdot \frac{\partial^{2} u_{f}}{\partial p_{i, j} \partial b_{i, j}}$ and $\frac{\partial^{2} u_{f}}{\partial b_{i, j} \partial p_{i, j}}$ are obtained identically as $W h_{i, S(i)}^{j}\left[(\ln 2) I_{i, j}\left(\mathbf{p}_{-i}\right)\left(\Omega+\gamma_{i, j}\right)\right]^{-1}-\mu_{i, j} h_{i}^{j}$. Since $\gamma_{i, j} \geq 0$, the sufficient condition of $\frac{\partial^{2} u_{f}}{\partial p_{i, j} \partial b_{i, j}}, \frac{\partial^{2} u_{f}}{\partial b_{i, j} \partial p_{i, j}} \leq 0$ is

$$
\mu_{i, j} \geq \frac{W h_{i, S(i)}^{j}}{(\ln 2) h_{i}^{j} \Omega I_{i, j}\left(\mathbf{p}_{-i}\right)} .
$$

To simplify the exposition, let $\mathbf{x}^{f} \triangleq\left[\mathbf{p}^{f}, \mathbf{b}^{f}\right]$ and $u_{f}\left(\mathbf{p}^{f}, \mathbf{b}^{f}, \gamma^{f}\right) \rightarrow u_{f}\left(\mathbf{x}^{f}, \gamma^{f}\right)$. Then, the Hessian matrix of $u_{f}$ with respect to $\mathbf{x}^{f}$ is negative semidefinite if $\mu_{i, j}$ for $\forall i \in \mathcal{F}_{u}^{f}, \forall j \in \mathcal{J}$ meets the inequality (22). Therefore, $u_{f}$ is concave, and thus quasi-concave in $\mathbf{x}_{f}$. Obviously, $u_{f}$ is continuous in $\mathbf{x}$.

Note that the inequality (22) contains the local information only and thus each femto BS can configure $\mu_{i, j}$ of their users to satisfy this condition.

$\operatorname{Prob}_{2}$ is analogous to the typical subcarrier-power resource allocation problem except the constraint $p_{i, j} \leq P_{i, j}$, and this problem has been studied extensively in the literature. Thus, we limit our concern to the case when $\sum_{j} b_{i, j} P_{i, j} \leq P_{\max }$ for $\forall i$, where femtocell interference mitigation becomes crucial for protecting macrocell services ( $\mathbb{P}^{f}$ becomes obsolete). We denote this modified problem as $\operatorname{Prob}_{2}^{\prime}$. For $\operatorname{Prob}_{2}^{\prime}$, we can design an instantly convergent control strategy as follows. The Lagrangian of $\operatorname{Prob}_{2}^{\prime}$ is

$$
\begin{aligned}
& =\sum_{i \in \mathcal{F}_{u}^{f}}^{L\left(\boldsymbol{\nu}, \mathbf{p}^{f}, \mathbf{b}^{f}\right)} \sum_{j \in \mathcal{J}} b_{i, j} W \log _{2}\left(1+\gamma_{i, j} / \Omega\right)-\sum_{i \in \mathcal{F}_{u}^{f}} \sum_{j \in \mathcal{J}} \mu_{i, j} h_{i}^{j} b_{i, j} p_{i, j} \\
& \quad+\sum_{j \in \mathcal{J}} \nu_{j}\left(1-\sum_{i \in \mathcal{F}_{u}^{f}} b_{i, j}\right)
\end{aligned}
$$

where $0 \preceq \mathbf{b}^{f} \preceq 1$ and $0 \preceq \mathbf{p}^{f} \preceq \mathbf{P}^{f} \cdot{ }^{13}$ Assume that $\mu_{i, j}$ meets the inequality (22) for $\forall i, j$. Then, $P r o b_{2}^{\prime}$ is a convex problem and thus any points that satisfy the Karush-KuhnTucker (KKT) conditions are optimal [31]. By exploiting one of the conditions, $\partial L / \partial p_{i, j}=0$, we obtain the optimal target SINR as

$$
\gamma_{i, j}^{*}=\max \left\{\left[\frac{W h_{i, S(i)}^{j}}{(\ln 2) I_{i, j}\left(\mathbf{p}_{-i}\right) \mu_{i, j} h_{i}^{j}}-\Omega\right]^{+}, \frac{h_{i, S(i)}^{j} P_{i, j}}{I_{i, j}\left(\mathbf{p}_{-i}\right)}\right\} .
$$

\footnotetext{
${ }^{13}$ The Lagrangian of $\mathrm{Prob}_{2}$ has an additional term for $\mathbb{P}^{f}$ : $\sum_{i \in \mathcal{F}_{u}^{f}} \xi_{i}\left(P_{\max }-\sum_{j \in \mathcal{J}} b_{i, j} p_{i, j}\right)$ where $\xi_{i}$ is a Lagrange multiplier.
} 
The sufficient condition that the TSINRC algorithm converges to $\gamma_{i, j}^{*}$ in Eq. (24) is presented below.

Proposition 6: $\mu_{i, j}=\lambda_{i, j} I_{i, j}^{-1}\left(\mathbf{p}_{-i}\right)$ is a sufficient condition of convergence of the TSINRC algorithm $\left(\lambda_{i, j}\right.$ is a constant).

If $\mu_{i, j}=\lambda_{i, j} I_{i, j}^{-1}\left(\mathbf{p}_{-i}\right), \gamma_{i, j}^{*}$ becomes independent of $\mathbf{p}_{-i}$, i.e., constant. Therefore, the TSINRC algorithm immediately converges to $\gamma_{i, j}^{*}$. Intuitively, $\mu_{i, j}=\lambda_{i, j} I_{i, j}^{-1}\left(\mathbf{p}_{-i}\right)$ means giving less penalty to those users suffering higher UL interference. Due to the nature of immediate convergence under this setting, the TSINRC algorithm can operate asynchronously with the MTXPC algorithm.

For resource block allocation, we can use another KKT condition $\partial L / \partial b_{i, j}=0$, whose solution is analogous to that of the typical problem, or a heuristic approach such as [32]. We omit the details of this.

\section{ITXPC Algorithm}

The TSINRC algorithm tracks the target SINR by controlling the instantaneous transmit power of a femtocell user. Since the algorithm is identical for each resource block, we drop the resource block index. The control system is simply presented as Eq. (21), which has a nonlinear relationship between components. For analytical tractability, we need to linearize the relationship. We first take the $\log$ of both sides of Eq. (21) and a log change of variables: $\widetilde{h}_{i, S(i)}=\log h_{i, S(i)}$, $\widetilde{p}_{i}=\log p_{i}, \widetilde{I}_{i}\left(\mathbf{p}_{-i}\right)=\log I_{i}\left(\mathbf{p}_{-i}\right)$ and, consequently, $\widetilde{\gamma}_{i}=$ $\log \gamma_{i}=\widetilde{h}_{i, S(i)}+\widetilde{p}_{i}-\widetilde{I}_{i}\left(\mathbf{p}_{-i}\right)$. Then, we replace the original error function $e_{i}=\gamma_{i}^{*}-\gamma_{i}$ defined in $\operatorname{Prob}_{3}$ with $\widetilde{e_{i}}=\widetilde{\gamma_{i}^{*}}-\widetilde{\gamma_{i}}$. It is clear that $\widetilde{e_{i}} \rightarrow 0$ if and only if $e_{i} \rightarrow 0$. Thus, the modified problem is valid.

Due to the bursty nature of the UL interference to femtocells, the controller should guarantee that the transmit power converges without overshoot or oscillation. We adopt an additive increase/decrease control for $\widetilde{p}_{i}: \widetilde{p}_{i}(t+1)=$ $\widetilde{p}_{i}(t)+K_{i} \widetilde{e}_{i}(t)$ where $K_{i}$ is constant. Then, the system transfer function is

$$
E(z)=\frac{z-1}{z+K_{i}-1}\left(\widetilde{\gamma_{i}^{*}}(z)+\widetilde{I}_{i}\left(\mathbf{p}_{-i}\right)(z)-\widetilde{h}_{i, S(i)}(z)\right) .
$$

An arbitrary step input generated at $t=0$ is expressed in the $z$ domain as $X_{0} z(z-1)^{-1}$ where $X_{0}$ is the step input amplitude. The time domain response to such a step input is then obtained by applying the inverse $z$-transform, as $\widetilde{e}_{i}(t)=X_{0}\left(1-K_{i}\right)^{t}$. Therefore, if $0<K_{i} \leq 1, \widetilde{e}_{i}$ converges to 0 without overshoot and oscillation, and so does $e_{i}$. It also implies that the ITXPC algorithm converges even with time-varying target SINR, UL interference and channel gain (even with a measurement error). Finally, the time-domain expression of the algorithm is given as

$$
p_{i}(t+1)=10^{\widetilde{p_{i}}(t+1)}=p_{i}(t) \cdot 10^{K_{i} \widetilde{e_{i}}(t)} .
$$

\section{NUMERICAL RESULTS}

To evaluate the effectiveness of CTRL, we first study the performance of each control loop in controllable environments. Then, we study multi-cell networks under more realistic conditions.
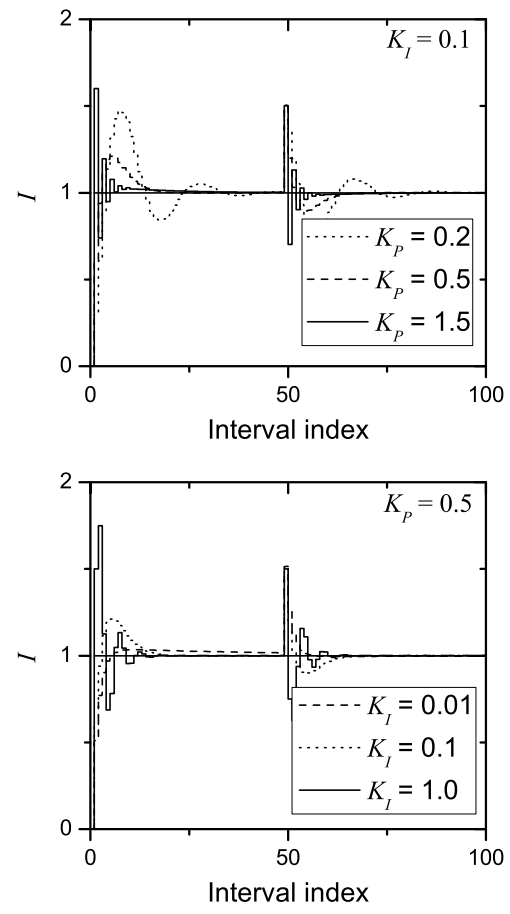

Fig. 5. Step response of the MTXPC loop

\section{A. Evaluation of Control Algorithms}

The MTXPC algorithm has two configuration parameters, $K_{P}$ and $K_{I}$, which determine its convergence behavior. Fig. 5 plots the response of the MTXPC loop to the step inputs $I_{t h}(0)=1$ and $I_{M}(50)=0.5$ when $Q=1$ and $d=0$. The MTXPC loop is shown to converge to the state $I=I_{t h}$ when $K_{P}$ and $K_{I}$ are within the range of Proposition 2. However, its transient behavior depends considerably on individual values. The left figure shows that too small $K_{P}(=0.2)$ results in oscillation. As $K_{P}$ increases, MTXPC becomes more responsive, but is accompanied with overshoot and oscillation when $K_{P}$ is too high $(=1.5)$. The right figure shows that, with too small $K_{I}$ $(=0.01)$, the convergence becomes slow while large $K_{I}(=1.0)$ leads to high overshoot as well as oscillation. Therefore, $K_{P}$ and $K_{I}$ need to be chosen carefully even within the stability region.

The error performance $E[|e|]$ (averaged over time) of the MTXPC loop against a time-varying macrocell load is plotted in Fig. 6 when $\left(K_{P}, K_{I}\right)=(0.5,0.1), Q=1$ and $d=5$. The macrocell load is modeled as a correlated Gaussian random variable with autocorrelation $\rho$. The figure shows that the error increases as the load changes faster (smaller $\rho$ ), the load has a higher deviation and the feedback delay gets larger. It is also shown that the MTXPC loop has a bounded error in the face of up to $100 \%$ overestimation of $Q$ and $d$. However, when the estimation error increases further, the loop becomes unstable and diverges since the Smith predictor fails to compensate the effect of delayed feedback (the result is omitted from the figure due to the difference in scale).

The left figure of Fig. 7 shows the cumulative distribution function (cdf) of the target SINR determined by the TSINRC loop when femtocells (each with a single user) are uniformly distributed within a circular macrocell. The cdf is shown to 

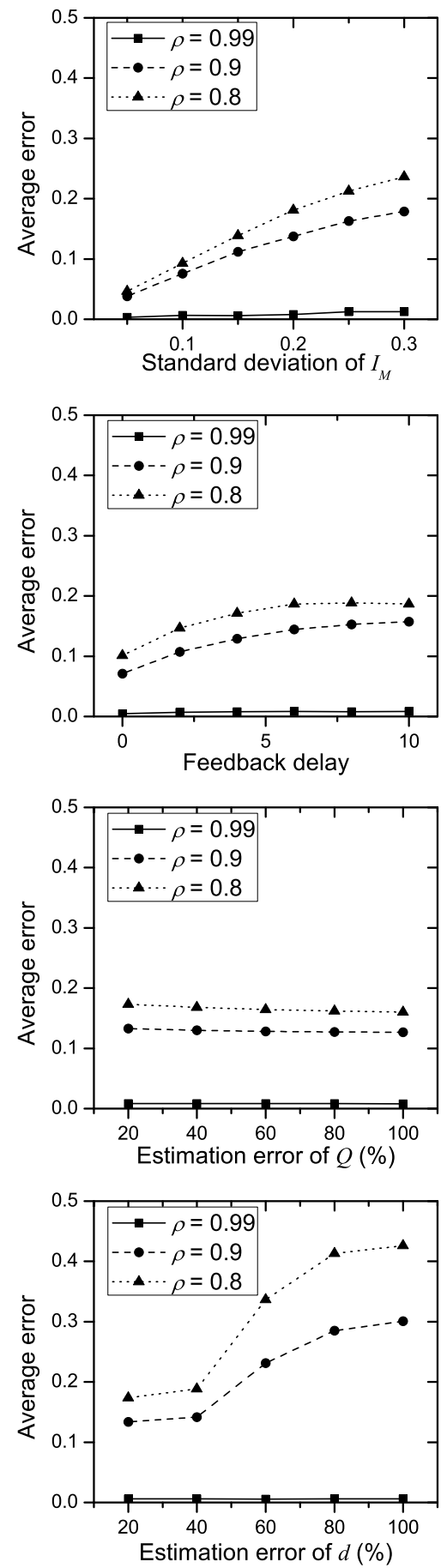

Fig. 6. Performance of the MTXPC loop under correlated Gaussian $I_{M}(Q$ $=1, d=5$ )

depend strongly on $\lambda_{i, j}$. Therefore, an inappropriate $\lambda_{i, j}$ value may force femtocell users to set too high target SINRs, causing high interference to macrocells. This configuration problem of the TSINRC loop is offset by the MTXPC loop. The right figure shows the error performance $E\left[\left|e_{i}\right|\right]$ (averaged over time) of the ITXPC loop while $K_{i}$ is varied. The UL interference is modeled as a two-state Markov chain where interference exists only in an active state. The idle-to-active transition probability $\pi_{0,1}$ is fixed at 0.5 . Then, the activeto-idle transition probability $\pi_{1,0}$ determines the burstiness
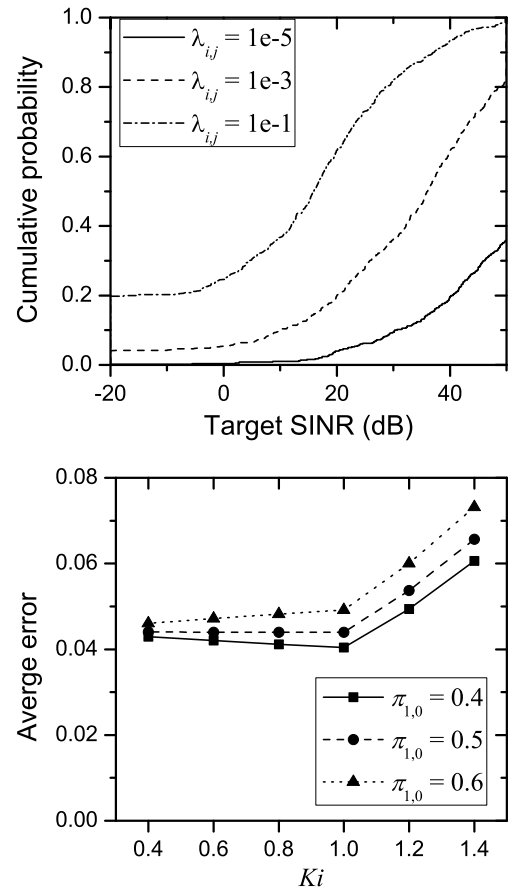

Fig. 7. Cumulative distribution of femtocell user's target SINR and the error performance of the ITXPC loop against bursty interference

of interference. $K_{i}=1$ means an immediate adjustment of transmit power to meet a target SINR in the next slot, thus achieving the highest performance against less bursty interference $\left(\pi_{1,0}=0.4\right)$, but lower performance against more bursty one $\left(\pi_{1,0}=0.6\right)$. When $K_{i}$ gets larger than 1 , the average error increases rapidly due to overshoot and oscillation.

\section{B. Multi-Cell Networks}

As a realistic communication environment, we consider a two-tier hexagonal cellular network comprised of 7 macrocells (each with a single sector). The radius of a macrocell is 500 $\mathrm{m}$ and 10 macrocell users are randomly distributed in each macrocell; the angle and the distance of each to the closest macro BS are randomly chosen with a uniform probability distribution. Femto BSs are randomly distributed within a macrocell and each of them serves a single user that is also randomly distributed within 50 meters from it (unless specified otherwise, the number of femtocells within a macrocell is 10). The path losses of a user to BSs are determined based on the ITU and COST231 models which are described as [34] [35]:

- macrocell user to macro BS (outdoor link):

$$
h=10^{4.9}\left(\frac{r}{1000}\right)^{4} f^{3} 10^{S / 10}
$$

- macrocell user to femto BS, femtocell user to macro BS (outdoor-to-indoor or indoor-to-outdoor link):

$$
h=10^{4.9}\left(\frac{r}{1000}\right)^{4} f^{3} 10^{S / 10} 10^{\left(L_{i}+L_{e}\right) / 10} ;
$$

- femtocell user to femto BS (indoor link):

$$
h=10^{3} r^{3.7} 10^{S / 10} 10^{L_{i} / 10},
$$


TABLE I

SIMULATION PARAMETERS

\begin{tabular}{l|l}
\hline Parameter & Value \\
\hline \hline Cellular layout & $\begin{array}{l}\text { Hexagonal layout with wrap- } \\
\text { around, } 7 \text { macro BSs, single cell } \\
\text { per macro BS }\end{array}$ \\
\hline Carrier frequency & $2.5 \mathrm{GHz}$ \\
\hline System bandwidth & $\begin{array}{l}2 \mathrm{MHz}(10 \text { resource blocks) for full } \\
\text { share }\end{array}$ \\
\hline Inter-site distance (ISD) & $500 \sqrt{3} \approx 866 \mathrm{~m}$ \\
\hline $\begin{array}{l}\text { Number of macrocell users } \\
\text { per macrocell }\end{array}$ & 10 \\
\hline $\begin{array}{l}\text { Number of femtocell users } \\
\text { per macrocell }\end{array}$ & 10 or 30 \\
\hline $\begin{array}{l}\text { Transmit power range of a } \\
\text { user }\end{array}$ & $-50 \sim 25 \mathrm{dBm}$ \\
\hline Fading & $\begin{array}{l}\text { Frequency-selective Rayleigh fad- } \\
\text { ing with a maximum doppler } \\
\text { spread of } 30 \mathrm{~Hz}\end{array}$ \\
\hline Shadowing & $\begin{array}{l}\text { Lognormal shadowing with a stan- } \\
\text { dard deviation of } 8 \mathrm{~dB}\end{array}$ \\
\hline $\begin{array}{l}\text { Minimum distance } \\
\text { between a macro BS and its } \\
\text { user }\end{array}$ & \begin{tabular}{l}
$30 \mathrm{~m}$ \\
\hline Thermal noise density
\end{tabular} \\
\hline User antenna gain & $-174 \mathrm{dBm} / \mathrm{Hz}$ \\
\hline Slot interval & $0 \mathrm{dBi}$ \\
\hline Scheduling algorithm & $1 \mathrm{~ms}$ \\
\hline Traffic type & $\begin{array}{l}\text { on/off with the Markov property on } \\
\text { a per-slot basis }\end{array}$ \\
\hline
\end{tabular}

where $r$ is the transmitter-receiver separation distance in meters; $f$ the carrier frequency in $\mathrm{MHz}$; $S$ the log-normal shadowing factor with a standard deviation of $8 \mathrm{~dB} ; L_{i}$ and $L_{e}$ are internal and external wall losses and set to 2 and $7 \mathrm{~dB}$, respectively, in our simulation.

Basically, both macro and femto BSs operate at the frequency band of $2.5 \mathrm{GHz}$ with fully-overlapping $2 \mathrm{MHz}$ bandwidth composed of 10 resource blocks. The frequency selective multipath channel is modeled to consist of six independent Rayleigh multipaths, with an exponentially decaying profile. A maximum doppler spread of $30 \mathrm{~Hz}$ is assumed. We define a slot as one unit of time and each slot interval as $1 \mathrm{~ms}$. User traffic arrivals (on/off) and resource scheduling including instantaneous transmit power change at a slot-level time scale. For resource block allocation, we use a scheme proposed by Wong et al. [33], which is a low-complexity version of Shen et al.'s [32]. First, the scheme by Wong et al. sequentially allocates each user the best resource block in terms of expected capacity. The remaining resource blocks are then allocated to users in the descending order of the ratio of expected capacity to the pre-determined user weight. In our simulation, all users within a cell are given an identical weight. The transmit power of a macrocell user is determined such that a given target SINR is immediately met, with which macrocell users' transmit power becomes highly dynamic compared to those by typical fixed-step power control schemes. This corresponds to $K_{i}=1$ in the ITXPC loop as explained in the previous subsection. Traffic arrival of a user in a slot is determined again by a two-state (active/idle) Markov chain. We fix the idle-toactive transition probability $\pi_{0,1}$ at 0.5 and, unless specified

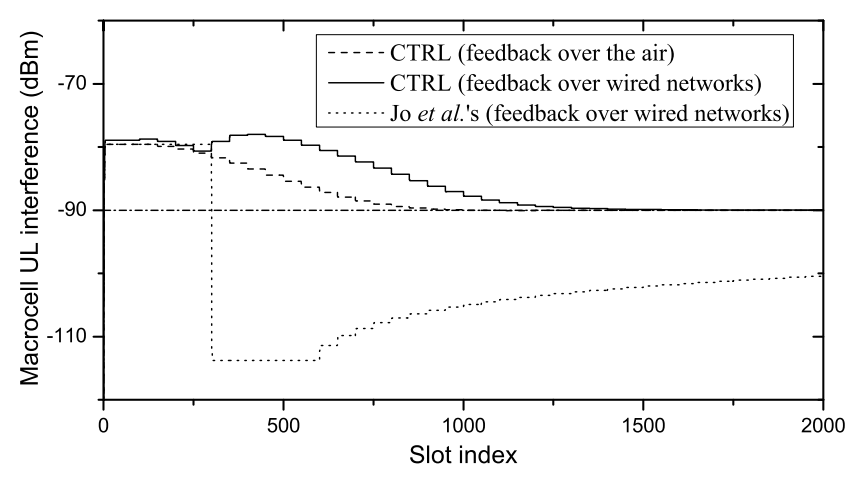

(a) $\pi_{1,0}=0$, w/o fading

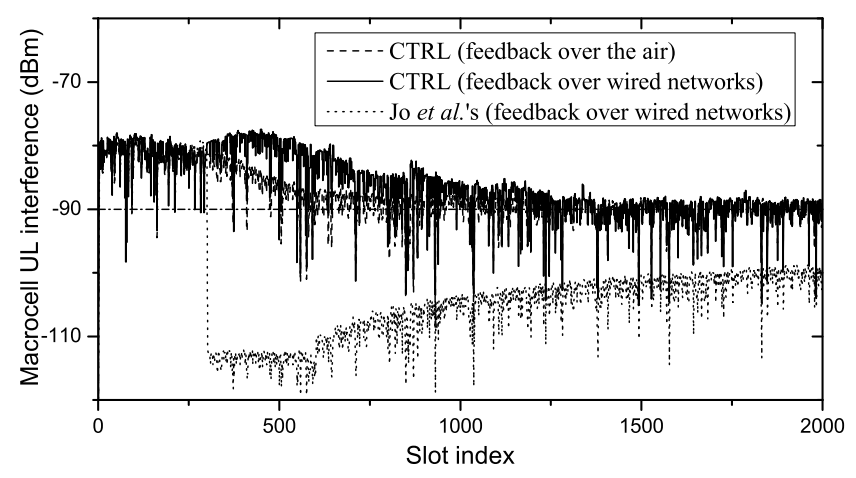

(b) $\pi_{1,0}=0.2, \mathrm{w} /$ fading

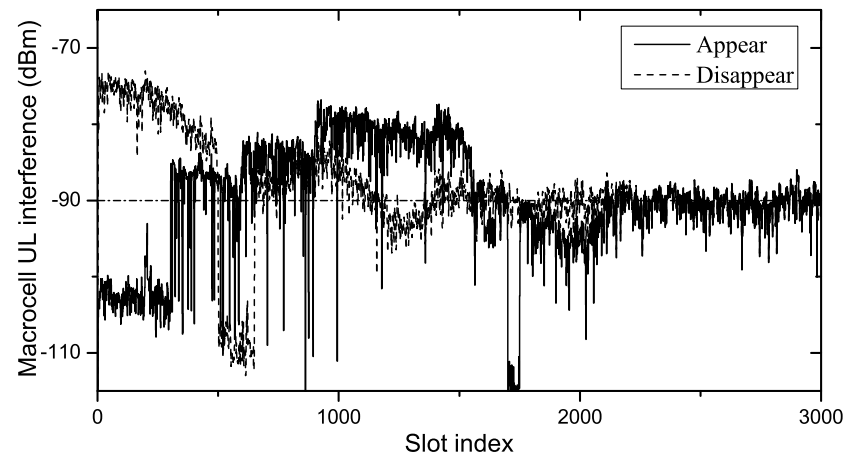

(c) Users appear/disapper. $\pi_{1,0}=0.2, \mathrm{w} /$ fading

Fig. 8. Time evolution of macrocell uplink interference for (a) static, (b) dynamic user transmission patterns and (c) the appearing/disappearing user scenario when $I_{t h}=-90 \mathrm{dBm}$

otherwise, the active-to-idle transition probability $\pi_{1,0}$ is set to 0.2 . The minimum and maximum transmit power levels of user devices are assumed to be -50 and $25 \mathrm{dBm}$, respectively. The feedback interval $T$ of the MTXPC loop is set to 50 slots. The load margin of each macrocell is measured at every slot and the average of measured samples during an entire feedback interval (i.e., the average of 50 samples) is fed back to femto BSs at the end of the interval. The feedback delay is either $5 T(d=5)$ to emulate the feedback over wired networks or $T(d=1)$ to emulate the feedback over the air. We set $q_{i}$ to 1 for $\forall i \in \mathcal{F}_{u}$ and $K_{i}$ to 0.8 . For the $Q$ estimation scheme, the forgetting factor $\lambda$ is set to 0.98 and $\hat{Q}$ is initialized to $10^{-5}$. $\alpha$ and $\beta$ are configured as 0.5 and 0.1 , respectively. $\mu_{i, j}$ is set to $10^{3}$ for $\forall i, j$. BER for femtocell users is $10^{-3}(\Omega \approx 3.3$ ). The simulation assumptions are summarized in Table I.

Fig. 8 shows the sample time evolutions of $I$ at the center macrocell with CTRL and with Jo et al.'s scheme when (a) 


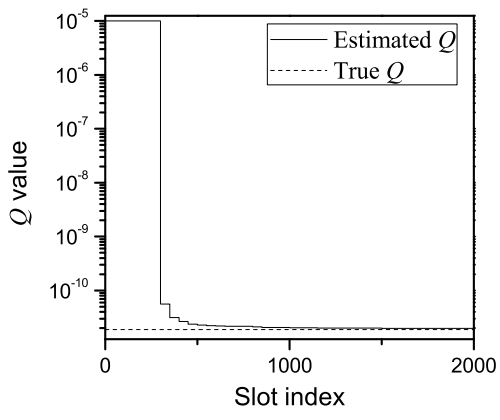

Fig. 9. Sample evolution of the estimated $Q$ value

$\pi_{1,0}=0$ without fading and (b) $\pi_{1,0}=0.2$ with fading. Ten femtocells are distributed in each macrocell and the desired convergence point $\left(I_{t h}\right)$ is configured as $-90 \mathrm{dBm}$. In both cases, CTRL converges to $I_{t h}$ while the one with feedback over the air $(d=1)$ achieves faster convergence without any oscillation than that with feedback over wired networks $(d=5)$, showing the result consistent with Fig. 6. Since $T=$ 50 slots, CTRL with feedback over wired networks converges within less than 40 feedback intervals. ${ }^{14}$ Under a dynamic user transmission pattern (b), I fluctuates somewhat, but the average is still close to $I_{t h}$ (that of the last 500 samples is $-89.9 \mathrm{dBm}$ ). However, Jo et al.'s scheme (with feedback over wired networks), even under the recommended system parameter setting requiring global information, ${ }^{15}$ fails to converge to the desired point and, moreover, accompanies an abrupt change of the UL interference level. For the remaining simulation study, the feedback delay of $5 T$ is considered to emulate feedback over wired networks. The evolution of the estimation of $Q$ for CTRL in this simulation run is shown in Fig. 9 where the proposed estimation scheme demonstrates the rapid convergence with high accuracy $\left(Q=1.88 e^{-11}\right.$ and the estimated value at the last interval is $\left.1.97 e^{-11}\right)$. The robustness of CTRL against dynamic user activities is investigated further in Fig. 10. Although CTRL achieves $I_{t h}$ in most of the observed slots, the variance increases as $\pi_{1,0}$ gets higher, which is somewhat inevitable due to the delayed feedback. We also consider a scenario in which users appear and disappear at different locations; we evaluate two different cases: (1) no femtocell users exist initially; a new femtocell user appears every 300 slots and (2) 20 femtocell users exist initially; one of the users disappears every 300 slots. The evaluation result is plotted in Fig. 8(c). In the figure, the macrocell's UL interference fluctuates more in both cases, but once converged, it is not highly affected by appearing/disappearing users.

The effects of the macrocell load threshold and the target SINR of macrocell users are explored in Fig. 11 and Fig. 12. The dominant trends observed in the figures are two folds: (1) the macrocell load threshold plays a role to control a tradeoff between macro- and femto-cell capacities; (2) an

\footnotetext{
${ }^{14}$ Each of the control loops has different convergence speeds; TSINRC is instantly convergent and ITXPC converges within several slots; MTXPC is the bottleneck of convergence since its feedback interval is much longer than the other two loops.

${ }^{15}$ To configure the system parameter of Jo et al.'s scheme $(\beta)$ as the authors recommended, each femto BS should have prior knowledge of the total number of active calls being served by femtocells.
}

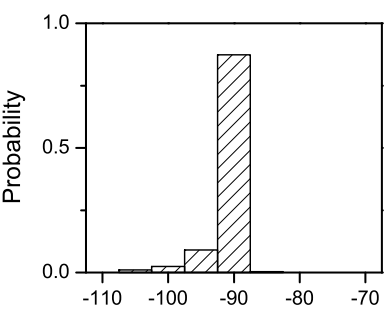

(a) $\pi_{1,0}=0.2$

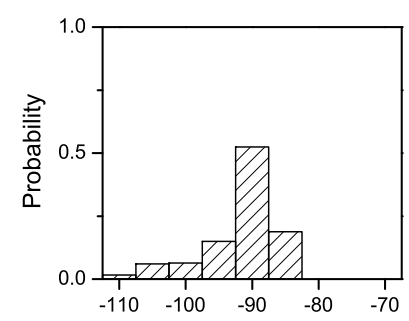

(c) $\pi_{1,0}=0.6$

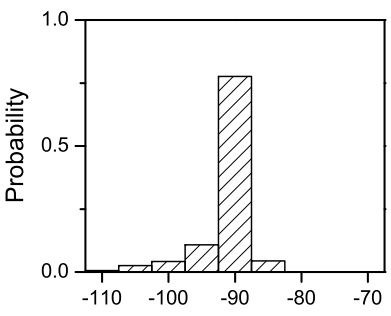

(b) $\pi_{1,0}=0.4$

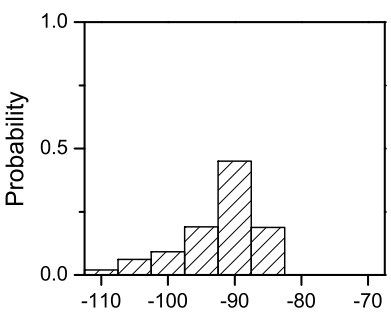

(d) $\pi_{1,0}=0.8$
Fig. 10. Probability distribution of macrocell uplink interference over time under various user activity patterns when $I_{t h}=-90 \mathrm{dBm}$

appropriate macrocell load threshold value for protecting the macrocell uplink depends on the target service quality of macrocell users. In Fig. 11 where the macrocell users' target SINR is set to $-5 \mathrm{~dB}$, if $I_{t h}=-100 \mathrm{dBm}, 70 \%$ of macrocell users achieve the target SINR. When $I_{t h}$ is increased to -90 $\mathrm{dBm}$, the achieved SINRs of macrocell users are generally degraded (only $20 \%$ of macrocell users achieve the target) and those of femtocell users are improved. This trend results from allowing femtocell users to use higher transmit power, i.e., to use more resources. The second trend is observed in Fig. 12. With (a) $I_{t h}=-100 \mathrm{dBm}$, more than half of macrocell users achieve the target SINRs of both -10 and $5 \mathrm{~dB}$. When (b) $I_{t h}=-90 \mathrm{dBm}$, around $40 \%$ of macrocell users with the target SINR of $-10 \mathrm{~dB}$ still achieve the target while most macrocell users with the target SINR of $-5 \mathrm{~dB}$ do not. Therefore, to provide better service quality to macrocell users, the resource usage of femtocell users should be limited more strictly by decreasing the macrocell load threshold. On the contrary, if lower service quality is required for macrocell users, a higher level of resource sharing can be allowed between macro- and femto-cells while protecting macrocell's uplink communications. In practice, $I_{t h}$ should be configured to provide room for unpredicted changes at the macrocell. On the other hand, femtocell users can have a pre-specified target SINR according to their services instead of being maximized by TSINRC. We also simulated such a scenario and plotted the result in Fig. 13 where lowering the target SINR of femtocell users is shown to reduce the interference to macrocell users, thus mitigating their performance degradation.

Fig. 11 also compares the cases with and without CTRL. For resource utilization within femtocells without CTRL, we assume that only the TSINRC and ITXPC loops are used. The leftmost figure shows that, with CTRL of $I_{t h}=-100 \mathrm{dBm}$, $70 \%$ of the macrocell users achieve their target SINR $(-5$ dB). Without CTRL, however, $80 \%$ of macrocell users achieve SINR below the target. These users are those who are far from their serving BSs and thus don't have enough room to increase 

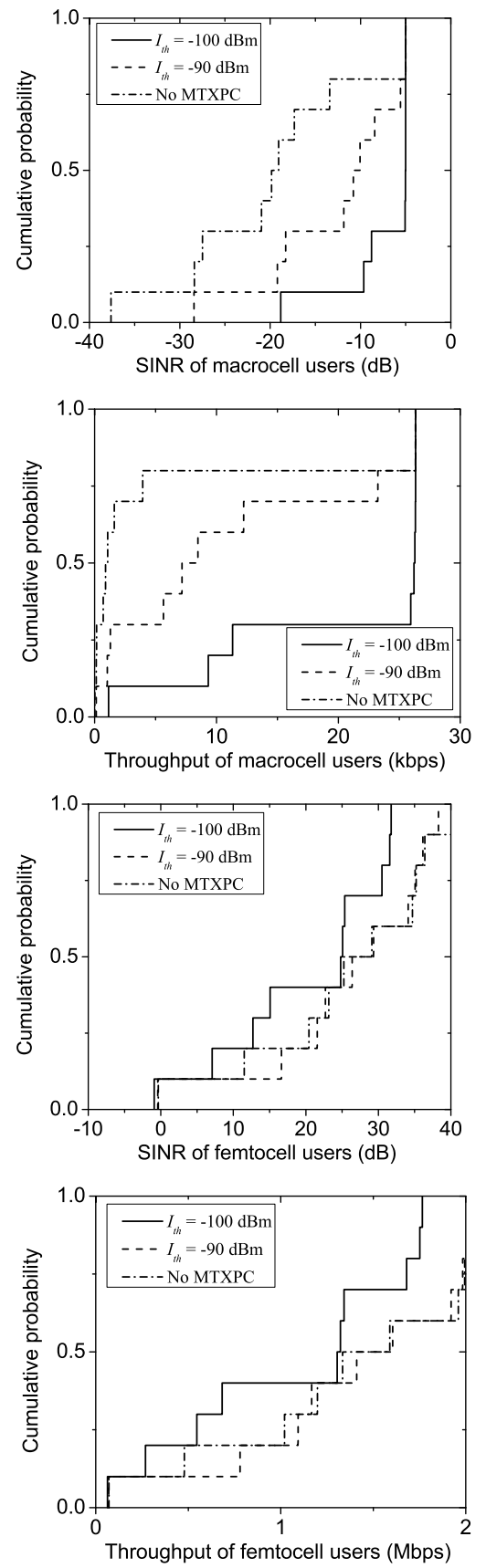

Fig. 11. Effect of the macrocell load threshold on achieved SINR and throughput when the target SINR of macrocell users is $-5 \mathrm{~dB}$

their transmit power against excessive UL interference. Due to this macrocell UL protection of CTRL, the macrocell UL throughput is improved by $165 \%$. It is shown in Fig. 14 that CTRL successfully protects the macrocell UL regardless of the increased number of femtocells per macrocell $(F / M)$; as $F / M$ increases from 10 to 30, the achieved SINRs of macrocell users with CTRL remain unchanged while, without CTRL, they are degraded considerably.

Finally, we investigate different frequency-allocation options. According to the degree of overlap between the system bandwidth of macrocells and that of femtocells, frequencyallocation options can be classified as dedicated, partiallyshared, and fully-shared. We assume that the total bandwidth

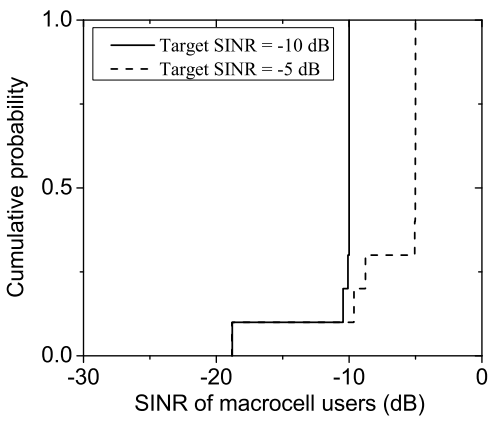

(a) $I_{t h}=-100 \mathrm{dBm}$

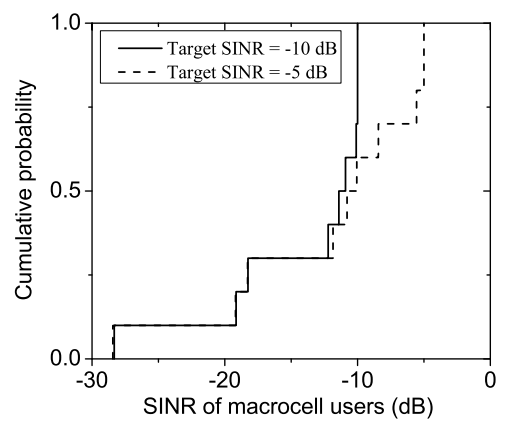

(b) $I_{t h}=-90 \mathrm{dBm}$

Fig. 12. Probability distribution of macrocell users' achieved SINR under different macrocell users' target SINR values
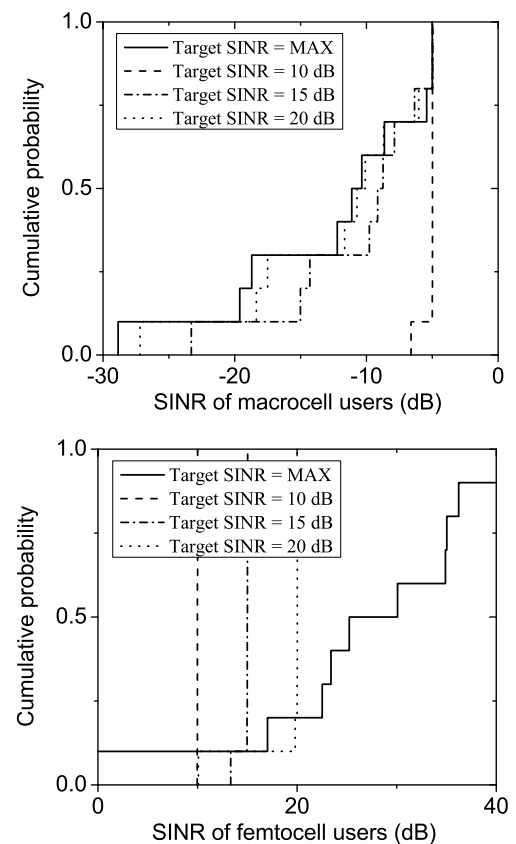

Fig. 13. Probability distribution of achieved SINR under different femtocell users' target SINR values

is fixed, and macro- and femto-cells are allocated the samesize bandwidth partitions. We can then identify a frequencyallocation option using the ratio of overlapping bandwidth to the system bandwidth allocated to a cell, which we call the share ratio. The dedicated frequency allocation is a $0 \%$ share ratio (null share for short) while the fully-shared allocation corresponds a 100\% share ratio (or full share for short). Our evaluation results for different share ratios are plotted in 


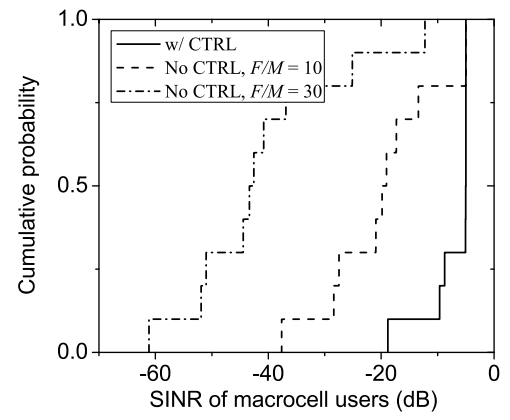

Fig. 14. Probability distribution of macrocell users' achieved SINR under various number of femtocell users when the target SINR of macrocell users is $-5 \mathrm{~dB}$ and $I_{t h}=-100 \mathrm{dBm}$

Fig. 15. The performance of macrocell users depend strongly on the presence of CTRL. With CTRL, the full share is the best for $70 \%$ of macrocell users since, by restricting the interference from femtocells, CTRL enables more macrocell users to benefit from the increased bandwidth. Without CTRL, however, the full share causes $70 \%$ of macrocell users to experience the worst performance because the performance loss due to the increased interference is larger than the performance gain from the increased bandwidth. On the other hand, femtocell users show a different trend: the full share is the best regardless of the use of CTRL. This is because femtocell users generally have much better channel quality than macrocell users, thus making their performance less affected by interference.

\section{CONCLUSION}

In this paper, we presented a novel femtocell management framework, called CTRL, for OFDMA-based cellular networks. CTRL is composed of three complementary control loops-MTXPC, TSINRC, and ITXPC-which protect the macrocell's uplink communication, coordinate resource usage of femtocells, and protect the femtocells' uplink communications. Based on the complementary interactions between these control loops, CTRL enables spatial reuse of channel resources within femtocells without degrading macrocell users' performance regardless of the number of femtocells operating in a macrocell. CTRL is designed to facilitate its deployment in existing cellular networks without requiring any change of the RRM of macro BSs. Moreover, CTRL ensures a distributed and self-organizing operation thanks to its estimation of the global femtocell status and convergence in the presence of time-varying and unpredictable environmental changes.

\section{ACKNOWLEDGEMENT}

This is a superset of the paper [36] we presented at ACM MobiCom 2010.

\section{APPENDIX A}

\section{ProOF OF Proposition 2}

Without loss of generality, in a discrete-time system, an arbitrary time-varying signal $X$ can be modeled as a piecewise constant model: $X(k)=\sum_{j=0}^{\infty} X_{0, j} \cdot 1\left(k-\tau_{j}\right)$ where $1(k)$ is the unitary step function, $X_{0, j} \in \mathbb{R}$, and $\tau_{j}$ is a time lag. When
$X(k)$ is input to a linear system, according to the superposition principle, the output becomes a linear combination of the system output of $X_{0, j} \cdot 1\left(k-\tau_{j}\right)$ for $\forall j$. Therefore, the problem for an arbitrary input is reduced to the problem for a step input with an arbitrary amplitude. In Fig. 4(b), the system transfer function is obtained as

$$
I(z)=\frac{\frac{C(z)}{z-1} Q}{1+\frac{C(z)}{z-1} Q} I_{t h}(z)+\frac{1}{1+\frac{C(z)}{z-1} Q}\left(I_{M}(z)-\epsilon(z)\right)
$$

and the system characteristic equation is $z^{2}+\left(Q\left(K_{P}+K_{I}\right)-\right.$ $2) z-Q K_{P}+1=0$. The system is stable if and only if all roots of the characteristic equation are inside the unit circle. According to the Jury test [23], the condition is met for the characteristic equation $C(z)=z^{2}+c_{1} z+c_{2}$ when $1-c_{2}^{2}>0$ and $1-c_{2}^{2}-\frac{\left(c_{1}-c_{1} c_{2}\right)^{2}}{1-c_{2}^{2}}>0$. From the first condition, $1-c_{2}^{2}=$ $Q K_{P}\left(2-Q K_{P}\right)>0$. If $Q K_{P}>0,0<K_{P}<2 / Q$. If $Q K_{P}<0, K_{P}>2 / Q$ and no feasible $K_{P}$ exists. From the second condition, $\frac{Q^{2} K_{P}^{2}\left(2-Q K_{P}\right)^{2}-Q^{2} K_{P}^{2}\left(Q\left(K_{P}+K_{I}\right)-2\right)^{2}}{Q K_{P}\left(2-Q K_{P}\right)}>0$ which reduces to $\left(2-Q K_{P}\right)^{2}>\left(Q\left(K_{P}+K_{I}\right)-2\right)^{2}$ since $0<K_{P}<2 / Q$, and then $0<K_{I}<4 / Q-2 K_{P}$.

\section{APPENDIX B \\ PROOF OF PROPOSITION 3}

The transfer function of $e(z)$ is obtained as

$$
\begin{aligned}
e(z) & =I_{t h}(z)-I_{M}(z)-I_{F}(z) \\
& =I_{t h}(z)-I_{M}(z)-\sum_{i \in \mathcal{F}_{u}} a_{i} h_{i} D_{i}(z) z^{-d} e(z)-\epsilon(z) \\
& =\left(1-\frac{\sum_{i \in \mathcal{F}_{u}} a_{i} h_{i} D_{i}^{*}(z) z^{-d}}{1+\sum_{i \in \mathcal{F}_{u}} a_{i} h_{i} D_{i}^{*}(z)}\right)\left(I_{t h}(z)-I_{M}(z)-\epsilon(z)\right)
\end{aligned}
$$

where the third equation follows from Eq. (8). Provided the MTXPC loop is stable (by using the parameters within the range obtained in Proposition 2), according to the final value theorem [25], the final value of $e$ in the time domain, denoted by $e_{\infty}$, is obtained as $e_{\infty}=\lim _{z \rightarrow 1}\left(1-z^{-1}\right) e(z)$. Since an arbitrary input of $I_{t h}(z)-I_{M}(z)-\epsilon(z)$ can be modeled as the sum of piecewise step functions, we only need to check convergence for a single step input. Applying the final value theorem to Eq. (28),

$$
\begin{aligned}
e_{\infty} & =\lim _{z \rightarrow 1}\left(1-z^{-1}\right)\left(1-\frac{\sum_{i \in \mathcal{F}_{u}} a_{i} h_{i} D_{i}^{*}(z) z^{-d}}{1+\sum_{i \in \mathcal{F}_{u}} a_{i} h_{i} D_{i}^{*}(z)}\right) \frac{z X_{0, j}}{z-1} \\
& =\lim _{z \rightarrow 1} X_{0, j}\left(1-\frac{\sum_{i \in \mathcal{F}_{u}} a_{i} h_{i} C_{i}(z) z^{-d}}{z-1+\sum_{i \in \mathcal{F}_{u}} a_{i} h_{i} C_{i}(z)}\right)=0
\end{aligned}
$$

where the third equation follows from $D_{i}^{*}(z)=\frac{C_{i}(z)}{z-1}$ in Eq. (14). While proving the proposition, we do not premise any specific type of $C(z)$. Thus, the above result is applicable to general types of $C(z)$ including Eq. (15).

\section{REFERENCES}

[1] 3G Home NodeB Study Item, 3GPP Technical Report 25.820, Rev. 8.2.0, Sep. 2008.

[2] FDD Home eNode B (HeNB) Radio Frequency (RF) requirements analysis, 3GPP Technical Report 36.921, Rev. 9.0.0, Mar. 2010.

[3] TDD Home eNode B (HeNB) Radio Frequency (RF) requirements analysis, 3GPP Technical Report 36.922, Rev. 9.1.0, Jun. 2010. 

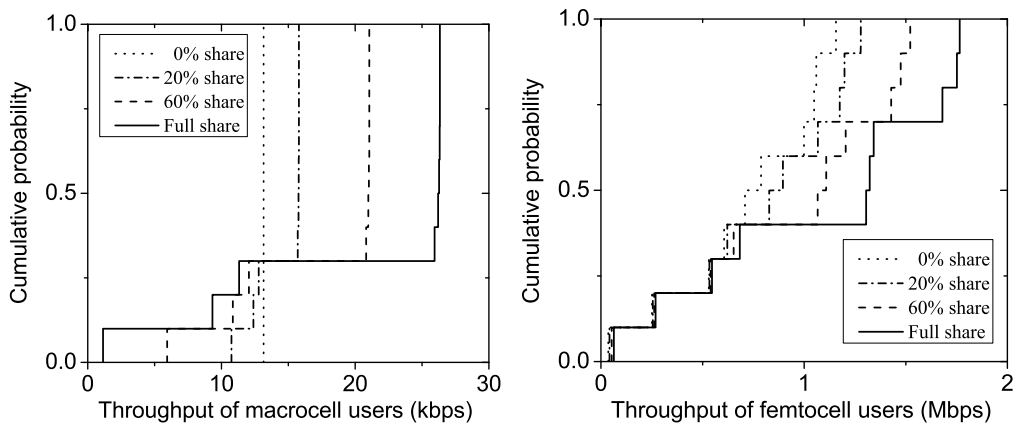

(a) With CTRL
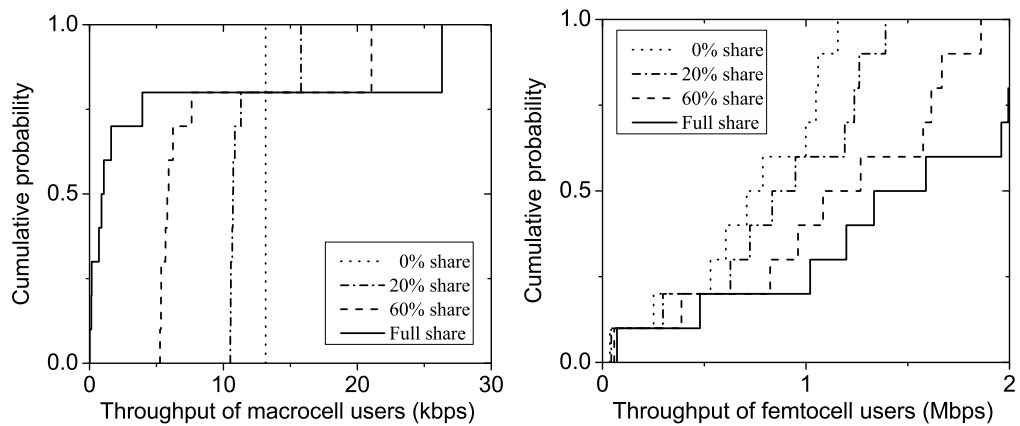

(b) Without CTRL

Fig. 15. Probability distribution of macrocell users' achieved SINR under various number of femtocell users when the target SINR of macrocell users is -5 $\mathrm{dB}$ and $I_{t h}=-100 \mathrm{dBm}$

[4] M. Yavuz, F. Meshkati, S. Nanda, A. Pokhariyal, N. Johnson, B. Raghothaman, and A. Richardson, "Interference management and performance analysis of UMTS/HSPA+ femtocells," IEEE Commun. Mag., vol. 47, no. 9, pp. 102 -109, Sep. 2009.

[5] V. Chandrasekhar and J. Andrews, "Uplink capacity and interference avoidance for two-tier femtocell networks," IEEE Trans. Wireless Commun., vol. 8, no. 7, pp. $3498-3509$, Jul. 2009.

[6] V. Chandrasekhar, J. Andrews, T. Muharemovic, Z. Shen, and A. Gatherer, "Power control in two-tier femtocell networks," IEEE Trans. Wireless Commun., vol. 8, no. 8, pp. 4316 -4328, Aug. 2009.

[7] H.-S. Jo, C. Mun, J. Moon, and J.-G. Yook, "Interference mitigation using uplink power control for two-tier femtocell networks," IEEE Trans. Wireless Commun., vol. 8, no. 10, pp. 4906 -4910, Oct. 2009.

[8] X. Wu, B. Murherjee, and D. Ghosal, "Hierarchical architectures in the third-generation cellular network," IEEE Wireless Commun., vol. 11, no. 3, pp. 62-71, 2004.

[9] L. Ho and H. Claussen, "Effects of user-deployed, co-channel femtocells on the call drop probability in a residential scenario," in IEEE 18th International Symposium on Personal, Indoor and Mobile Radio Communications, 2007 (PIMRC'07), Sep. 2007, pp. 1 -5.

[10] H. Ji and C.-Y. Huang, "Non-cooperative uplink power control in cellular radio systems," Wireless Networks, vol. 4, no. 3, pp. 233-240, Mar. 1998.

[11] D. Lopez-Perez, A. Valcarce, G. de la Roche, and J. Zhang, "OFDMA femtocells: A roadmap on interference avoidance," IEEE Commun. Mag., vol. 47, no. 9, pp. 41-48, Sep. 2009.

[12] K. Sundaresan and S. Rangarajan, "Efficient resource management in OFDMA femto cells," in MobiHoc '09: Proceedings of the tenth ACM international symposium on Mobile ad hoc networking and computing. New York, NY, USA: ACM, 2009, pp. 33-42.

[13] J. Jin and B. Li, "Cooperative resource management in cognitive WiMAX with femto cells," in Proc. IEEE INFOCOM, Mar. 2010, pp. $1-9$.

[14] D. Lopez-Perez, A. Valcarce, A. Ladanyi, G. de la Roche, and J. Zhang, "Intracell handover for interference and handover mitigation in OFDMA two-tier macrocell-femtocell networks," EURASIP Journal on Wireless Communications and Networking, vol. 2010, 2010, article ID 142629, doi:10.1155/2010/142629.

[15] V. Chandrasekhar and J. Andrews, "Spectrum allocation in tiered cellular networks," IEEE Trans. Commun., vol. 57, no. 10, pp. 3059 -3068, Oct. 2009.
[16] K. Yeung and S. Nanda, "Channel management in microcell/macrocell cellular radio systems," IEEE Trans. Veh. Technol., vol. 45, no. 4, pp. $601-612$, Nov. 1996.

[17] X. Lagrange, "Multitier cell design," IEEE Commun. Mag., vol. 35, no. 8, pp. $60-64$, Aug. 1997.

[18] Evolved Universal Terrestrial Radio Access (E-UTRA); Physical Channels and Modulation, 3GPP Technical Specification 36.211, Rev. 9.1.0, Mar. 2010.

[19] L. Ding, M. S. El-Hennawey, and R. A. Goubran, "Nonintrusive measurement of echo-path parameters in VoIP environments," IEEE Trans. Instrum. Meas., vol. 55, no. 6, pp. 2062-2071, 2006.

[20] O. Gurewitz, I. Cidon, and M. Sidi, "One-way delay estimation using network-wide measurements," IEEE Trans. Inf. Theory, vol. 52, no. 6, pp. 2710-2724, 2006.

[21] H. Wicaksana, S. Ting, C. Ho, W. Chin, and Y. Guan, "AF two-path half duplex relaying with inter-relay self interference cancellation: diversity analysis and its improvement," IEEE Trans. Wireless Commun., vol. 8, no. 9, pp. $4720-4729$, Sep. 2009.

[22] Radio Resource Control (RRC) Protocol Specification (Release 8), 3GPP Technical Specification 25.331, Rev. 8.4.0, Sep. 2008.

[23] K. J. Astrom and B. Wittenmark, Computer-Controlled Systems: Theory and Design. Prentice Hall, 1996.

[24] J. E. Normey-Rico and E. F. Camacho, Control of Dead-time Processes. Springer, 2007.

[25] G. F. Franklin, J. D. Powell, and A. Emami-Naeini, Feedback Control of Dynamic Systems. Prentice Hall, 1994.

[26] K. J. Astrom and B. Wittenmark, Adaptive Control. Prentice Hall, 1994.

[27] J. Nash, "Non-cooperative games," The Annals of Mathematics, vol. 54, no. 2, pp. 286-295, 1951. [Online]. Available: http://www.jstor.org/stable/1969529

[28] H. Kwon and B. G. Lee, "Distributed resource allocation through noncooperative game approach in multi-cell OFDMA systems," in Proc. IEEE Int. Conf. Communications ICC '06, vol. 9, 2006, pp. 4345-4350.

[29] S. T. Chung and A. J. Goldsmith, "Degrees of freedom in adaptive modulation: a unified view," IEEE Trans. Commun., vol. 49, no. 9, pp. 1561-1571, 2001.

[30] J. F. Nash, "Equilibrium points in n-person games," in Proc. Natl. Acad. Sci. U.S.A., vol. 36, no. 1, Jan. 1950, pp. 48-49.

[31] L. V. Stephen Boyd, Convex Op. Cambridge University Press, 2004.

[32] Z. Shen, J. G. Andrews, and B. L. Evans, "Adaptive resource allocation 
in multiuser OFDM systems with proportional rate constraints," IEEE Trans. Wireless Commun., vol. 4, no. 6, pp. 2726-2737, 2005.

[33] I. C. Wong, Z. Shen, B. L. Evans, and J. G. Andrews, "A low complexity algorithm for proportional resource allocation in OFDMA systems," in Proc. IEEE Workshop Signal Processing Systems SIPS 2004, 2004, pp. $1-6$.

[34] Guidelines for evaluation of radio transmission technologies for IMT2000, ITU-R Rec M.1225, Feb. 1997.

[35] C. A. 231, "Digital mobile radio towards future generation systems, final report," European Communities, Tech. Rep. EUR 18957, 1999.

[36] J.-H. Yun and K. G. Shin, "CTRL: A self-organizing femtocell management architecture for co-channel deployment," in Proc. ACM International conference on Mobile computing and networking (MobiCom'10), Chicago, IL, USA, Sep. 2010, pp. 61-72.

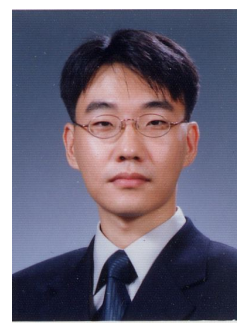

Ji-Hoon Yun is currently an assistant professor in the Department of Computer Software Engineering, Kumoh National Institute of Technology (KIT), Gumi, Korea. Before joining KIT in September 2010, he was with the Real-Time Computing Laboratory (RTCL) at The University of Michigan, Ann Arbor, U.S.A. as a postdoctoral researcher. He was a senior engineer at the Telecommunication Systems Division, Samsung Electronics, Suwon, Korea from 2007 to 2009. He received the B.S. degree in Electrical Engineering from Seoul National University (SNU), Seoul, Korea in 2000, and both the M.S. and Ph.D. degrees in Electrical Engineering and Computer Science from SNU in 2002 and 2007, respectively. His current research focuses on wireless networks and efficient computing of mobile devices.

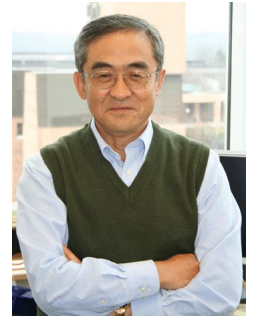

Kang G. Shin is the Kevin \& Nancy O'Connor Professor of Computer Science in the Department of Electrical Engineering and Computer Science, University of Michigan, Ann Arbor. His current research focuses on computing systems and networks as well as on embedded real-time and cyber-physical systems, all with emphasis on timeliness, security, and dependability.

$\mathrm{He}$ has supervised the completion of $67 \mathrm{PhDs}$, and authored/coauthored about 750 technical articles, one a textbook and more than 20 patents or invention disclosures, and received numerous best paper awards, including the Best Paper Awards from the 2010 and 2000 USENIX Annual Technical Conferences, the IEEE Communications Society William R. Bennett Prize Paper Award in 2003, an Outstanding IEEE Transactions of Automatic Control Paper Award in 1987. He has also received several institutional awards, including the Research Excellence Award in 1989, Outstanding Achievement Award in 1999, Distinguished Faculty Achievement Award in 2001, and Stephen Attwood Award in 2004 from The University of Michigan (the highest honor bestowed to Michigan Engineering faculty); a Distinguished Alumni Award of the College of Engineering, Seoul National University in 2002; 2003 IEEE RTC Technical Achievement Award; and 2006 Ho-Am Prize in Engineering (the highest honor bestowed to Korean-origin engineers).

He has chaired several major conferences, including 2009 ACM MobiCom, 2008 IEEE SECON, 2005 ACM/USENIX MobiSys, 2000 IEEE RTAS, and 1987 IEEE RTSS. He is the fellow of both IEEE and ACM, and served on editorial boards, including IEEE TPDS and ACM Transactions on Embedded Systems. 